\title{
AN ERROR ESTIMATE FOR FINITE VOLUME METHODS FOR MULTIDIMENSIONAL CONSERVATION LAWS
}

\author{
BERNARDO COCKBURN, FRÉDÉRIC COQUEL, AND PHILIPPE LEFLOCH
}

\begin{abstract}
In this paper, an $L^{\infty}\left(L^{1}\right)$-error estimate for a class of finite volume methods for the approximation of scalar multidimensional conservation laws is obtained. These methods can be formally high-order accurate and are defined on general triangulations. The error is proven to be of order $h^{1 / 4}$, where $h$ represents the "size" of the mesh, via an extension of Kuznetsov approximation theory for which no estimate of the total variation and of the modulus of continuity in time are needed. The result is new even for the finite volume method constructed from monotone numerical flux functions.
\end{abstract}

\section{INTRODUCTION}

In this paper, a modification of the Kuznetsov approximation theory for multidimensional scalar conservation laws $[28,29]$ is obtained which is then used to obtain an $L^{\infty}\left(L^{1}\right)$-error estimate for the class of monotone finite volume methods (which are at most first-order accurate only), as well as for high-order schemes constructed upon some of them. We consider the Cauchy problem for a multidimensional scalar conservation law ([26, 30, 31, 51]):

$$
\begin{array}{ll}
\partial_{t} u+\operatorname{div} f(u)=0 & \text { in } \mathbf{R}_{+} \times \mathbf{R}^{d}, \\
u(0)=u_{0} & \text { on } \mathbf{R}^{d},
\end{array}
$$

where the flux function $f: \mathbf{R} \rightarrow \mathbf{R}^{d}$ is assumed to be smooth and the initial data $u_{0}$ is taken to be in the space $L^{\infty}\left(\mathbf{R}^{d}\right) \cap B V\left(\mathbf{R}^{d}\right)$ of bounded functions of bounded variation in $\mathbf{R}^{d}$. In [28, 41], error estimates are obtained for approximations $u^{h}$ to the solution of $(1.1)-(1.2)$ which satisfy the following properties (for each $T>0$, and some constant $C=C(T)>0$ ):

Received by the editor March 19, 1991 and, in revised form, December 17, 1992 and September 21, 1993.

1991 Mathematics Subject Classification. Primary 65M60, 65N30, 35L65.

Key words and phrases. Multidimensional conservation law, discontinuous solution, finite volume method, error estimate.

Work of the first author was partially supported by the NSF grant DMS-91-03997.

Work of the third author was partially supported by NSF grants DMS-88-06731 and DMS-9209326. 


$$
\begin{aligned}
\text { (i) } & \left\|u^{h}\right\|_{L^{\infty}\left((0, T) \times \mathbf{R}^{d}\right)} \leq C, \\
\text { (ii) } & \left\|u^{h}(t)-u^{h}\left(t^{\prime}\right)\right\|_{L^{1}\left(\mathbf{R}^{d}\right)} \leq C\left(\left|t-t^{\prime}\right|+h\right), \\
\text { (iii) } & \left\|u^{h}\right\|_{L^{\infty}\left(0, T ; B V\left(\mathbf{R}^{d}\right)\right)} \leq C, \\
\text { (iv) } & E^{h} \leq C h,
\end{aligned}
$$

where $E^{h}$ represents the term of entropy dissipation associated with $u^{h}$. In this paper, we obtain an error estimate for numerical schemes that need not satisfy the above conditions. Among them are the monotone schemes defined on general triangulations (for which no proof of the estimate (iii) is available) and the finite volume methods obtained from some monotone schemes by the so-called $h^{\gamma}$-antidiffusion technique (for which none of the above properties hold). The main idea in this paper is that the error estimate follows from a weak estimate for the uniform norm and a weak upper bound of the entropy dissipation of the approximations, i.e., estimates of the form (for each $T>0$ ):

$$
\begin{aligned}
\text { (i) } & \left\|u^{h}\right\|_{L^{\infty}\left((0, T) \times \mathbf{R}^{d}\right)} \leq \mathscr{O}\left(h^{-\nu_{1}}\right) \\
\text { (iv) } & E^{h} \leq \mathscr{O}\left(h^{\nu_{2}}\right)
\end{aligned}
$$

for some numbers $\nu_{1}$ and $\nu_{2}$ in $(0,1]$. (Compare with the setting due to Tadmor for one-dimensional problems using compensated compactness arguments [47].)

Let us recall that Kuznetzov [28, 29] was the first to obtain an error estimate for the monotone schemes for (1.1) after the pionnering works by Kružkov [26, 27], and Volpert [51]. Using grids which are Cartesian products of uniform onedimensional partitions, Kuznetsov proved that the error $\left\|u(t)-u^{h}(t)\right\|_{L^{1}\left(\mathbf{R}^{d}\right)}$ between the exact solution $u$ and the approximate solution $u^{h}$ is $\mathscr{O}\left(h^{1 / 2}\right)$, as $h$ goes to zero (uniformly for $t$ in $(0, T)$ ). Sanders [41, 42] (see also Osher and Sanders [39]) later proved that the same rate of the $L^{\infty}\left(L^{1}\right)$-error holds for monotone schemes constructed by using two-point monotone fluxes on nonuniform Cartesian grids. Error estimates for other numerical schemes that also satisfy the properties (i) to (iv) (or a refined version of them) have been obtained by Lucier in [34, 35, and 36]. Hoff and Smoller [22] first proved that the error in Glimm's scheme is $\mathscr{O}\left(h^{1 / 6} \ln h\right)$. We also refer to Chern [4] who proves an error estimate for Glimm's scheme applied to systems of conservation laws. Cf. also Johnson and Szepessy [25] who treat a finite element method for one-dimensional systems. For the so-called quasi-monotone schemes, [5, $6,7]$, the estimate of the entropy dissipation (iv) does not hold. Cockburn [5] modified the Kuznetsov approximation theory and proved that the $L^{\infty}\left(L^{1}\right)$ error for these schemes is $\mathscr{O}\left(h^{\gamma / 2}\right)$ for some number $\gamma \in(0,1]$. Recently, Tadmor [47] (also [37]) proposed a general framework to obtain error estimates for one-dimensional scalar conservation laws. His method allowed him to get error estimates for several first-order and second-order methods. The present work presents the first result on error estimates for a multidimensional problem with general triangulations.

For a background on the analysis and the convergence of difference schemes, we refer to the works by Harten, Hyman, and Lax [20], and Harten, Lax, and van Leer [21]. Observe that Crandall and Majda proved in [16] a general theorem of 
convergence of the monotone schemes. See also Goodman and LeVeque [19], Hou and LeFloch [23], LeFloch and Liu [33], Osher [38], Osher and Tadmor [40], and Tadmor [46].

Szepessy [44, 45], for the streamline diffusion method, and Coquel and LeFloch $[13,14,15]$, for high-order difference schemes, proved the convergence of numerical schemes without appealing to a uniform BV estimate (iii). To do so, the framework of DiPerna's measure-valued solutions [18] was used. The importance of deriving an estimate of the rate of entropy dissipation for difference schemes was emphasized by Coquel and LeFloch in [14]. Chen, Du, and Tadmor [1] next used [18] in their analysis of spectral methods. This approach is related to the compensated compactness technique, for which we refer the reader to DiPerna [17], as well as [2, 3, 24, and 47], and the references therein.

The present paper continues the work initiated in [9], where the convergence of the monotone finite volume methods was proven. The idea that prompted this work is the following. On the one hand, DiPerna's [18] uniqueness result for (1.1), (1.2) in the class of measure-valued solutions was based on Kružkov's techniques [26]. On the other hand, Kuznetsov approximation theory [28] is also based on Kružkov's approach. It is then reasonable to expect that by using Kuznetsov's theory, the measure-valued approach could be bypassed. In this paper we prove that this is indeed the case. Our technique of proof in this paper involves more work than in [9], but has the advantage of providing not only the convergence, but also an estimate of the error.

We consider here a large class of numerical schemes, which can be highorder accurate, and derive an error estimate from a suitable modification of Kuznetsov's approach. We do not need an estimate of the total variation like (iii), nor an estimate of the modulus of continuity in time like (ii). For monotone schemes, although the modulus of continuity in time is uniformly bounded, as a consequence of the $L^{1}$-contraction property, no proof of the boundedness on the total variation is available. The $L^{1}$-contraction property does not hold for high-order accurate schemes, and so an approximation theory that does not require any estimate on the modulus of continuity in time is essential. Our proof is based on a formulation of the discrete entropy inequalities and on the so-called entropy dissipation estimate that were derived in [9]. Note that, in order to make use of these inequalities, it is necessary to introduce suitably chosen piecewise constant test functions adapted from the original paper by Kuznetsov to our case. This is due to the fact that, for general triangulations, the property of invariance by translation is lost. The result in this paper can be easily extended to the Runge-Kutta type discretizations introduced in $[10,11$, and 12].

The paper is organized as follows. In $\S 2$, we state the hypotheses on the triangulations, define the class of schemes under consideration, and state the error estimate (see Theorem 2.1). In $\S 3$, we give the proof of Theorem 2.1 , decomposed into five subsections: (a) the basic inequality, (b) estimating the lack of symmetry of the entropy, (c) estimating the entropy production associated with the exact solution, (d) estimating the entropy production associated with the approximate solution, (e) completion of the proof of Theorem 2.1. Finally, in $\S 4$, we prove that high-order accurate schemes built upon monotone schemes satisfying sharp entropy inequalities beiong to the class of numerical schemes for which Theorem 2.1 holds. 


\section{Statement of the MAin Result}

Let $t_{n}=n \tau, n=0,1, \ldots$, be a uniform mesh for the discretization of $\mathbf{R}_{+}$ and, for each $h>0$, let $\mathscr{T}_{h}$ be a triangulation of $\mathbf{R}^{d}$ composed of nonoverlapping, and nonempty polyhedra. As is usual in the finite element approach, we assume that, if two distinct elements $K_{1}$ and $K_{2}$ in $\mathscr{T}_{h}$ have a nonempty intersection, say $I$, then either $I$ is a face of both $K_{1}$ and $K_{2}$, or $I$ has Hausdorff dimension less than $d-1$. The set of faces of a polyhedron $K$ is denoted by $\partial K$, and, for each face on $K, N_{e, K} \in \mathbf{R}^{d}$ represents the outward unit normal vector to the face $e$. Given a face $e$ of $K$, then $K_{e}$ is the unique polyhedron which shares the same face $e$ with $K$. The volume of $K$ and the $(d-1)$-measure of $e$ are denoted by $|K|$ and $|e|$, respectively. Without loss of generality, we can assume that

$$
h=\sup _{K \in \mathscr{T}_{h}} h_{K}<+\infty,
$$

where $h_{K}$ is the exterior diameter of a polyhedron. The perimeter of $K$ is defined by $p_{K}=\sum_{e \in \partial K}|e|$. The interior diameter of an element $K$ is denoted by $\rho_{K}$.

We assume the following conditions on $\tau$ and $\mathscr{T}_{h}$ :

$$
\frac{h}{\gamma} \leq \tau \leq \gamma h,
$$

and

$$
\frac{h_{K}}{\rho_{K}} \leq \sigma \quad \text { for all } K \in \mathscr{T}_{h},
$$

where $\gamma \geq 1$ and $\sigma>0$ are independent of $h$. We observe that (2.2) implies

$$
\frac{1}{\mu} \leq \frac{p_{K} h_{K}}{|K|} \leq \mu \quad \text { for all } K \in \mathscr{T}_{h}
$$

for some $\mu>0$.

The finite volume methods under consideration produce a function, say $u^{h}$, defined from $\mathbf{R}_{+}$to $L^{1}\left(\mathbf{R}^{d}\right)$, which is right-continuous in time and piecewise constant, namely,

$$
u^{h}(t, x)=u_{K}^{n} \quad \text { for }(t, x) \in\left[t_{n}, t_{n+1}\right) \times K .
$$

We define the contants $u_{K}^{n}$ as follows. First of all, we have to approximate the exact flux function of equation (1.1). Let us introduce a family of numerical flux terms $g_{e, K}^{n}$, given for each face $e$ of each polyhedron $K$ of $\mathscr{T}_{h}$, that are locally Lipschitz continuous functions depending upon a finite number (fixed for all the triangulations) of values $u_{K^{\prime}}^{n}$ (e.g., $u_{K}^{n}$ and $u_{K_{e}}^{n}$ for a two-point numerical flux), and satisfy the following two properties:

$$
\text { conservation: } \quad g_{e, K}^{n}+g_{e, K_{e}}^{n}=0,
$$

and, for each real number $u$,

$$
\text { consistency: } \quad u_{K^{\prime}}^{n}=u \text { for all } K^{\prime} \Longrightarrow g_{e, K}^{n}=N_{e, K} \cdot f(u) .
$$


The notation $z \cdot z^{\prime}$ and $|z|$ will be used for the Euclidean scalar product and the Euclidean norm of vectors in $\mathbf{R}^{d}$, respectively. Let us also denote by $\mathbf{P}_{h}$ a projection operator (for example, the $L^{2}$-projection operator) into the space of functions of the variable $x$ which are constant in each $K \in \mathscr{T}_{h}$.

We are now ready to introduce the high-order accurate finite volume method. By definition, the approximate solutions $u^{h}$ have the form (2.4) with

$$
u^{h}(0)=\mathbf{P}_{h}\left(u_{0}\right),
$$

and for $n=0,1,2, \ldots$ and all $K \in \mathscr{T}_{h}$

$$
u_{K}^{n+1}=u_{K}^{n}-\frac{\tau}{|K|} \sum_{e \in \partial K}|e| g_{e, K}^{n} \text {. }
$$

To guarantee the stability of the method, the Courant-Friedrichs-Levy number $\nu$ must be less than 1:

$$
\nu=\sup \frac{\tau p_{K}}{|K|}\left|N_{e, K} \cdot \frac{d f}{d u}(u)\right| \leq 1,
$$

where the supremum is taken over all elements $K$, all faces $e$, and all values $u$ under consideration.

Since we want to recover the entropy solution to problem $(1.1)-(1.2)$, the numerical flux in $(2.6 \mathrm{~b})$ must satisfy a discrete version of the entropy inequality. Following an idea due to Tadmor [46], and following Coquel and LeFloch [15], we express $u_{K}^{n+1}$ as a convex combination of one-dimensional discrete operators:

$$
u_{K}^{n+1}=\frac{1}{p_{K}} \sum_{e \in \partial K} u_{K, e}^{n+1}|e|,
$$

where

$$
u_{K, e}^{n+1}=u_{K}^{n}-\frac{\tau p_{K}}{|K|}\left\{g_{e, K}^{n}-N_{e, K} \cdot f\left(u_{K}^{n}\right)\right\} .
$$

A discrete entropy inequality for $u_{K}^{n+1}$ will follow if each of the values $u_{K, e}^{n+1}$, $e \in \partial K$, satisfies an (essentially one-dimensional) discrete entropy inequality.

Following Kružkov and Kuznetsov, we focus our attention on the set of inequalities associated with the so-called Kružkov entropies. We recall that the classical Kružkov entropies form a one-parameter family of entropy-entropy flux pairs for equation (1.1):

$$
U(v, w)=|v-w|, \quad F(v, w)=\operatorname{sgn}(w-v)(f(w)-f(v)) \quad(v, w) \in \mathbf{R}^{2} .
$$

Observe that $(U, F)$ is an entropy with respect to one variable, when the other is kept constant. Moreover, $(U, F)$ is symmetric with respect to $(v, w)$.

Instead of working directly with (2.9), since this is difficult with high-order schemes, cf. the entropy inequality (2.15) below, it will be convenient to consider the following regularization:

$$
U_{M}(v, w)= \begin{cases}|w-v|-1 / 2 M & \text { for }|w-v| \geq 1 / M, \\ M|w-v|^{2} / 2 & \text { for }|w-v| \leq 1 / M\end{cases}
$$

As $M$ tends to infinity, we recover the Kružkov entropies. The function $U_{M}$ is strictly convex with respect to both variables, and satisfies

$$
\left\|\frac{d U_{M}}{d u}\right\|_{L^{\infty}(\mathbf{R})}=1, \quad\left\|\frac{d^{2} U_{M}}{d u^{2}}\right\|_{L^{\infty}(\mathbf{R})}=M .
$$


We view $U_{M}$ as an entropy function with respect to the first variable. The flux associated with this entropy is given by

$$
F_{M}(v, w)=\int_{w}^{v} \frac{\partial U_{M}}{\partial s}(s, w) f^{\prime}(s) d s .
$$

Notice that, unlike $F$, the function $F_{M}$, unfortunately, is no longer symmetric:

$$
F_{M}(v, w)-F_{M}(w, v)=\int_{w}^{v}\left(\frac{\partial U_{M}}{\partial s}(s, v)+\frac{\partial U_{M}}{\partial s}(s, w)\right) f^{\prime}(s) d s
$$

and in particular is not an entropy flux with respect to its second argument. This is a difficulty when applying Kuznetsov theory. However, we are able to overcome this difficulty by using the fact that the difference

$$
\partial_{v}\left(F_{M}(v, w)-F_{M}(w, v)\right)=\int_{w}^{v} \frac{\partial^{2} U_{M}}{\partial s^{2}}(s, v)\left[f^{\prime}(v)-f^{\prime}(s)\right] d s
$$

is of order $1 / M$; see the proof of Lemma 3.2.

We assume that, for each $M>0$ and each $c$, and for each $e$ and $K$, there are numerical entropy flux terms $G_{e, K}^{n}$, which are locally Lipschitz continuous functions depending on a finite number of mesh values. We also assume that they are conservative and consistent with the entropy flux $F_{M}(\cdot, c)$, that is,

$$
\begin{gathered}
G_{e, K}^{n}+G_{e, K_{e}}^{n}=0, \\
u_{K^{\prime}}^{n}=u \text { for all } K^{\prime} \Longrightarrow G_{e, K}^{n}=N_{e, K} \cdot F_{M}(u, c),
\end{gathered}
$$

and for which a discrete entropy inequality holds $[5,14]$ :

$$
\begin{aligned}
& U_{M}\left(u_{K, e}^{n+1}, c\right)-U_{M}\left(u_{K}^{n}, c\right)+\frac{\tau p_{K}}{|K|}\left\{G_{e, K}^{n}-N_{e, K} \cdot F_{M}\left(u_{K}^{n}, c\right)\right\} \\
& \quad \leq \frac{\tau p_{K}}{|K|} a_{K, e}^{n} \frac{\partial U_{M}}{\partial v}\left(v_{K, e}^{n}, c\right) .
\end{aligned}
$$

The quantities $a_{K, e}^{n}$ and $v_{K, e}^{n}$ in the right-hand side of (2.15) are assumed to satisfy for all time $T$ the following estimate:

$$
\sum_{n \tau \leq T} \sum_{\substack{K \in \mathcal{G}_{h} \\ e \in \theta K}}\left|a_{K, e}^{n}\right|\left|v_{K, e}^{n}-v_{K_{e}, e}^{n} \| e\right| \tau \leq C_{1} h^{\alpha}
$$

for some $\alpha>0$ and $C_{1}=C_{1}(T)>0$. In addition, the terms $a_{K, e}^{n}$ are assumed to obey the following conservation property, similar to $(2.5 \mathrm{a})$ :

$$
a_{K, e}^{n}+a_{K_{e}, e}^{n}=0 .
$$

Finally, we assume that the amplitude of the approximation does not grow faster than $h^{-\beta}$ for some $\beta \in[0,1)$; specifically for all time $T>0$,

$$
\left\|u^{h}\right\|_{L^{\infty}\left([0, T] \times \mathbf{R}^{d}\right)} \leq\left\|u_{0}\right\|_{L^{\infty}\left(\mathbf{R}^{d}\right)}+C_{2} h^{-\beta}
$$

for some constant $C_{2}=C_{2}(T)>0$. For instance, for the monotone schemes built up with two-point monotone fluxes, we can take $a_{K, e}^{n} \equiv 0$, so that $C_{1}=$ $C_{2}=0$, and take $U_{M}$ as the Kružkov entropies, i.e., $1 / M=0$ in (2.15). Finally, we assume that the flux function is at most quadratic at infinity, in the following sense:

$$
\limsup _{|w| \rightarrow \infty} \frac{1}{|w|}\left|\frac{d f}{d u}(w)\right|<\infty, \quad \limsup _{|w| \rightarrow \infty}\left|\frac{d^{2} f}{d u^{2}}(w)\right|<\infty .
$$

(Obviously, (2.19) is irrelevant in the case that $\left\|u^{h}\right\|_{L^{\infty}}$ is uniformly bounded.) 
We now state the main result whose proof is given in the next section.

Theorem 2.1. Let $u$ be the entropy solution to problem (1.1)-(1.2) with an initial data $u_{0} \in B V\left(\mathbf{R}^{d}\right) \cap L^{\infty}\left(\mathbf{R}^{d}\right)$. Let $u^{h}$ be the approximate solution defined by the (possibly high-order accurate) finite volume method (2.4), (2.6). We assume that the conditions (2.1)-(2.3), (2.5), (2.7) and (2.15)-(2.19) hold. Then for all time $t$ and any positive scalars $A$ and $B$ with $B$ large enough (i.e., larger than the maximum speed in the scheme), the following error estimate holds:

$$
\begin{aligned}
\left\|u^{h}(t)-u(t)\right\|_{L^{1}\left(\Delta_{t}\right) \leq} & C_{0} h^{1 / 4}\left\|u_{0}\right\|_{L^{2}\left(\mathbf{R}^{d}\right)}^{1 / 2}+C_{0}^{\prime} h^{1 / 2}\left(\left\|u_{0}\right\|_{L^{\infty}\left(\mathbf{R}^{d}\right)}+T V\left(u_{0}\right)\right) \\
& +C_{0}^{\prime \prime} h^{\min \{\alpha, 1-\beta\} / 2}
\end{aligned}
$$

where $\Delta_{t}=\{|x| \leq A-t B\}$ is a domain of influence/dependence, $C_{0}$ and $C_{0}^{\prime}$ depend on $t$ and $A$, and $C_{0}^{\prime}$ depends on $t \cdot T V\left(u_{0}\right)$. Furthermore, $C_{0}^{\prime \prime}=0$ if $C_{1}=C_{2}=0$.

For monotone schemes, $C_{0}^{\prime \prime}=0$, Theorem 2.1 shows that the rate of convergence of the finite volume method is $\mathscr{O}\left(h^{1 / 4}\right)$ when measured in the $L^{1}$ norm. This rate seems to be optimal, at least with the technique developed in this paper. For the monotone schemes defined in Cartesian uniform grids, considered by Kuznetsov [28], or in nonuniform Cartesian grids considered by Sanders [41], the rate of convergence is $h^{1 / 2}$. However, in this latter case, the scheme is uniformly bounded in the total variation norm (TVB), and the original Kuznetsov technique applies. Our result extends the error estimate in [28] and [41] to schemes that are not necessarily stable in the BV norm. Note that our basic assumption is the set of inequalities (2.15). Our result is new even with $a_{K, e}^{n}=0$.

Following Coquel and LeFloch [14] in the case of Cartesian meshes, one easily sees that Theorem 2.1 applies to the class of schemes based on the so-called corrected antidiffusive flux technique. Theorem 2.1 indeed applies to the highorder accurate schemes (defined on quasi-uniform triangulations) built with the so-called $h^{\gamma}$-antidiffusion method upon monotone schemes that satisfy sharp entropy inequalities, in the sense of [14]. In $\S 4$, we prove that these schemes satisfy the entropy inequality (2.15), and the estimate (2.16) with $\alpha=1 / p$, for some $p \geq 2$; moreover, we show that the upper bound (2.18) with $\beta=0$ holds when the triangulations are quasi-uniform. By Theorem 2.1 , this implies that these schemes converge with a rate of $\mathscr{O}\left(h^{1 / 2 p}\right)$. A recent work by Vila [50] also treats the extension to more general high-order explicit or implicit schemes.

We emphasize that the uniformity of the time-discretization has been assumed for the sake of simplicity. Theorem 2.1 remains true for nonuniform time-discretizations satisfying the standard restrictions. Theorem 2.1 also extends to more general space triangulations that do not satisfy the properties (2.1)-(2.2) and are not necessarily composed of polyhedra, but admit a refinement made of $d$-dimensional polyhedra that satisfies (2.1)-(2.2). The triangulations $\mathscr{T}_{h}$ themselves could also depend upon $t_{n}$, as is necessary for mesh refinement techniques.

\section{Proof of the main Result}

In this section we give the proof of Theorem 2.1. Our proof is based on a suitable modification of the classical Kuznetsov approximation result. 
Proposition 3.1, derived below at the end of the first subsection, yields a basic inequality for $u-u^{h}$ measured in the $L^{1}$ norm which:

(1) does not involve the modulus of continuity in time of the approximate solutions,

(2) is based on the regularization (2.10) of the Kružkov entropies, and

(3) involves a piecewise constant approximation of the standard Kuznetsov test function, which is necessary to apply the discrete entropy inequalities derived in [9].

This basic inequality involves two kinds of terms: one measures the lack of symmetry in the regularized version of the Kružkov entropies; two other terms measure the entropy production relative to the functions $u$ and $u^{h}$, respectively.

In Proposition 3.2, we use (2.13) to prove that the error term due to the lack of symmetry of the regularized entropy flux defined by $(2.12)$ is at most $O(1) / M$. In Propositions 3.3 and 3.4, we estimate the entropy production associated with the exact solution $u$ and the approximate solution $u^{h}$, respectively. Finally, in a last subsection, we complete the proof of Theorem 2.1.

For simplicity in the presentation, and in the rest of the paper, the initial data $u_{0}$ in (1.2) is assumed to have compact support. All the estimates below have straightforward extensions to trapezoid-shaped domains (as stated in the main theorem), which hold for arbitrary initial data. We denote by $\Omega^{h}(t)$ the union of the supports of the functions $u$ and $u^{h}$ at time $t$, which clearly satisfies $\left|\Omega^{h}(t)\right|=O(1) t^{d}$ for large $t$. For convenience in the presentation, we fix a bounded time interval $[0, T]$, such that $\tau n_{T}=T$, for some integer $n_{T}$, and we then estimate $\left\|u^{h}(T)-u(T)\right\|_{L^{1}}$.

We point out that, in the case of monotone schemes (on arbitrary triangulations), the Kružkov entropies can be used (i.e., $1 / M$ can be taken to be zero). In that case, the $L^{1}$-contraction property does provide an estimate of the modulus of continuity in time, and it can be proven that

$$
\left\|u^{h}(t)-u^{h}\left(t^{\prime}\right)\right\|_{L^{1}\left(\mathbf{R}^{d}\right)} \leq T V\left(\mathbf{P}_{h}\left(u_{0}\right)\right)\left(\left|t-t^{\prime}\right|+\tau\right),
$$

where $T V\left(\mathbf{P}_{h}\left(u_{0}\right)\right)$ remains uniformly bounded (see, for a proof, [8]), owing to the assumptions (2.1) and (2.2).

3.1. The basic inequality. Our first objective is to derive a generalization of Kuznetsov's approximation inequality for $\int_{\mathbf{R}^{d}}\left|u^{h}(T, x)-u(T, x)\right| d x$, i.e., Proposition 3.1 below. We are going to work with a special class of test functions $\phi$ we now define. Let $\epsilon_{0}$ and $\epsilon$ be arbitrary positive real numbers. Let $\omega$ : $\mathbf{R} \rightarrow \mathbf{R}$ be a smooth nonnegative even function with unit mass, and support in $[-1,1]$, and, for any positive number $\gamma$, let us set $\omega_{\gamma}(s)=\frac{1}{\gamma} \omega(s / \gamma)$ for all $s$ in $\mathbf{R}$. We consider the function $\phi$ defined by

$$
\phi\left(t, x ; t^{\prime}, x^{\prime}\right)=\omega_{\epsilon_{0}}\left(t-t^{\prime}\right) \psi_{\epsilon}\left(x-x^{\prime}\right), \quad \psi_{\epsilon}\left(x-x^{\prime}\right)=\prod_{i=1}^{d} \omega_{\epsilon}\left(x_{i}-x_{i}^{\prime}\right) .
$$

To simplify the notation, and if there is no risk of confusion, we will often drop either the variable $(t, x)$, or $\left(t^{\prime}, x^{\prime}\right)$, or both. We observe that the support of $\phi$ shrinks to the "line" $\left\{(t, x)=\left(t^{\prime}, x^{\prime}\right)\right\}$ as $\epsilon_{0}, \epsilon \rightarrow 0$. We shall use the 
notation $Q_{T}=(0, T) \times \mathbf{R}^{d}$, or $Q_{T}^{\prime}=(0, T) \times \mathbf{R}^{d}$ if $\left(t^{\prime}, x^{\prime}\right)$ are the relevant variables.

To use the entropy inequalities in [9], we must introduce the following piecewise constant approximations of the functions $\omega_{\epsilon_{0}}\left(t-t^{\prime}\right)$ and $\psi_{\epsilon}\left(x-x^{\prime}\right)$ :

$$
\begin{aligned}
& \tilde{\omega}_{\epsilon_{0}}\left(t ; t^{\prime}\right)=\omega_{\epsilon_{0}}\left(t_{n+1}-t^{\prime}\right) \quad \text { for } t \in\left[t_{n}, t_{n+1}\right), \\
& \tilde{\omega}_{\epsilon_{0}}^{\prime}\left(t ; t^{\prime}\right)=\omega_{\epsilon_{0}}\left(t-t_{n+1}^{\prime}\right) \quad \text { for } t^{\prime} \in\left[t_{n}^{\prime}, t_{n+1}^{\prime}\right),
\end{aligned}
$$

and

$$
\begin{aligned}
& \tilde{\psi}_{\epsilon}\left(x ; x^{\prime}\right)=\psi_{\epsilon, K}\left(x^{\prime}\right)=\frac{1}{p_{K}} \sum_{e \in \partial K} \int_{e} \psi_{\epsilon}\left(y-x^{\prime}\right) d \Gamma(y), \\
& x \in K, x^{\prime} \in \mathbf{R}^{d}, K \in \mathscr{T}_{h}, \\
& \tilde{\psi}_{\epsilon}^{\prime}\left(x ; x^{\prime}\right)=\psi_{\epsilon, K^{\prime}}^{\prime}(x)=\frac{1}{p_{K^{\prime}}} \sum_{e^{\prime} \in \partial K^{\prime}} \int_{e^{\prime}} \psi_{\epsilon}\left(x-y^{\prime}\right) d \Gamma\left(y^{\prime}\right), \\
& x \in \mathbf{R}^{d}, x^{\prime} \in K^{\prime}, K^{\prime} \in \mathscr{T}_{h} .
\end{aligned}
$$

Next we define the corresponding approximations $\partial_{t}^{h} \phi, \partial_{t^{\prime}}^{h} \phi, \nabla^{h} \phi$, and $\nabla^{h^{\prime}} \phi$ of the exact time derivative and space gradients of the test function $\phi$, respectively:

$$
\begin{aligned}
& \partial_{t}^{h} \phi\left(t, x ; t^{\prime}, x^{\prime}\right)=\tilde{\psi}_{\epsilon}\left(x ; x^{\prime}\right) \partial_{t} \omega_{\epsilon_{0}}\left(t-t^{\prime}\right), \\
& \partial_{t^{\prime}}^{h} \phi\left(t, x ; t^{\prime}, x^{\prime}\right)=-\tilde{\psi}_{\epsilon}^{\prime}\left(x ; x^{\prime}\right) \partial_{t} \omega_{\epsilon_{0}}\left(t-t^{\prime}\right),
\end{aligned}
$$

and

$$
\begin{aligned}
& \nabla^{h} \phi\left(t, x ; t^{\prime}, x^{\prime}\right)=\tilde{\omega}_{\epsilon_{0}}\left(t ; t^{\prime}\right) \nabla \psi_{\epsilon}\left(x-x^{\prime}\right), \\
& \nabla^{h^{\prime}} \phi\left(t, x ; t^{\prime}, x^{\prime}\right)=-\tilde{\omega}_{\epsilon_{0}}^{\prime}\left(t ; t^{\prime}\right) \nabla \psi_{\epsilon}\left(x-x^{\prime}\right) .
\end{aligned}
$$

Similarly, it will be convenient to introduce a piecewise constant approximation of the exact solution $u$ :

$$
\tilde{u}(t, x)=u\left(t_{n}, x\right) \text { for } t \in\left[t_{n}, t_{n+1}\right) \text {. }
$$

The quantities $u(t, x), \tilde{u}(t, x)$, and $u^{h}\left(t^{\prime}, x^{\prime}\right)$ will be abbreviated as $u, \tilde{u}$, and $u^{h}$, respectively.

For definiteness, we consider $u$ and $u^{h}$ as right-continuous functions from $[0, T)$ to $L^{1}\left(\mathbf{R}^{d}\right)$ whose limits from the left exist on $(0, T]$. The function $u$ represents the entropy solution to problem $(1.1)-(1.2)$, while $u^{h}$ denotes the piecewise constant approximate solution (2.4) given by the scheme (2.6). We start our derivation by introducing the approximate entropy dissipation form $E_{\epsilon_{0}, \epsilon}^{h}\left(u, u^{h}\right)$ as follows:

$$
E_{\epsilon_{0}, \epsilon}^{h}\left(u, u^{h}\right)=\int_{Q_{T}^{\prime}} \Theta_{\epsilon_{0}, \epsilon}^{h}\left(u, u^{h}\left(t^{\prime}, x^{\prime}\right) ; t^{\prime}, x^{\prime}\right) d x^{\prime} d t^{\prime}
$$


where, for all constants $c$,

$$
\begin{aligned}
\boldsymbol{\Theta}_{\epsilon_{0}, \epsilon}^{h}( & \left.u, c ; t^{\prime}, x^{\prime}\right) \\
= & -\int_{Q_{T}}\left\{U_{M}(\tilde{u}, c) \partial_{t}^{h} \phi\left(t^{\prime}, x^{\prime}\right)+F_{M}(u, c) \cdot \nabla^{h} \phi\left(t^{\prime}, x^{\prime}\right)\right\} d x d t \\
& -\int_{\mathbf{R}^{d}} U_{M}(u(0, x), c) \phi\left(0, x ; t^{\prime}, x^{\prime}\right) d x \\
& +\int_{\mathbf{R}^{d}} U_{M}(u(T, x), c) \phi\left(T, x ; t^{\prime}, x^{\prime}\right) d x .
\end{aligned}
$$

The term $\boldsymbol{\Theta}_{\epsilon_{0}, \epsilon}^{h}\left(u, c ; t^{\prime}, x^{\prime}\right)$ is a measure of the entropy dissipation associated with the entropy solution $u$ : it is basically nonpositive; Proposition 3.3 below will give a precise statement. Observe that $\tilde{u}$ defined by (3.6) appears in the first term of the right-hand side of $(3.7 b)$ : this is due to the fact that the time derivative of $u^{h}$ needs special treatment, as was observed in [9]. Setting $\tilde{u}^{h}=u^{h}$ (which is a natural definition in view of the definition (3.6) of $\tilde{u}$ and the fact that $u^{h}$ is piecewise constant), we can define $E_{\epsilon_{0}, \epsilon}^{h}\left(u^{h}, u\right)$ and $\boldsymbol{\theta}_{\epsilon_{0}, \epsilon}^{h}\left(c, u^{h} ; t, x\right)$ by the same formulas.

Following Kuznetsov, and using the fact that $U_{M}$ is a symmetric function, we have the following identity:

$$
R_{\epsilon_{0}, \epsilon}^{h}\left(u, u^{h}\right)=S_{\epsilon_{0}, \epsilon}^{h}\left(u, u^{h}\right)+E_{\epsilon_{0}, \epsilon}^{h}\left(u, u^{h}\right)+E_{\epsilon_{0}, \epsilon}^{h}\left(u^{h}, u\right)
$$

where

$$
\begin{aligned}
R_{\epsilon_{0}, \epsilon}^{h}\left(u, u^{h}\right)= & \int_{Q_{T}} \int_{\mathbf{R}^{d}} U_{M}\left(u^{h}\left(T, x^{\prime}\right), u(t, x)\right) \phi\left(t, x ; T, x^{\prime}\right) d x^{\prime} d x d t \\
& +\int_{Q_{T}^{\prime}} \int_{\mathbf{R}^{d}} U_{M}\left(u^{h}\left(t^{\prime}, x^{\prime}\right), u(T, x)\right) \phi\left(T, x ; t^{\prime}, x^{\prime}\right) d x d x^{\prime} d t^{\prime} \\
& -\int_{Q_{T}} \int_{\mathbf{R}^{d}} U_{M}\left(u^{h}\left(0, x^{\prime}\right), u(t, x)\right) \phi\left(t, x ; 0, x^{\prime}\right) d x^{\prime} d x d t \\
& -\int_{Q_{T}^{\prime}} \int_{\mathbf{R}^{d}} U_{M}\left(u^{h}\left(t^{\prime}, x^{\prime}\right), u(0, x)\right) \phi\left(0, x ; t^{\prime}, x^{\prime}\right) d x d x^{\prime} d t^{\prime},
\end{aligned}
$$

and

$$
\begin{aligned}
S_{\epsilon_{0}, \epsilon}^{h}( & \left.u, u^{h}\right) \\
= & \int_{Q_{T}} \int_{Q_{T}^{\prime}}\left\{F_{M}\left(u, u^{h}\right) \tilde{\omega}_{\epsilon_{0}}-F_{M}\left(u^{h}, u\right) \tilde{\omega}_{\epsilon_{0}}^{\prime}\right\} \cdot \nabla \psi_{\epsilon} d x^{\prime} d t^{\prime} d x d t \\
& +\int_{Q_{T}} \int_{Q_{T}^{\prime}}\left\{U_{M}\left(\tilde{u}, u^{h}\right) \tilde{\psi}_{\epsilon}-U_{M}\left(u, u^{h}\right) \tilde{\psi}_{\epsilon}^{\prime}\right\} \partial_{t} \omega_{\epsilon_{0}} d x^{\prime} d t^{\prime} d x d t .
\end{aligned}
$$

Since, as $\epsilon, \epsilon_{0} \rightarrow 0$, the term $R_{\epsilon_{0}, \epsilon}^{h}\left(u, u^{h}\right)$ is expected to converge to

$$
\int_{\mathbf{R}^{d}} U_{M}\left(u^{h}(T, x), u(T, x)\right) d x-\int_{\mathbf{R}^{d}} U_{M}\left(u^{h}(0, x), u(0, x)\right) d x,
$$

it will be called the error term. Note that $S_{\epsilon_{0}, \epsilon}^{h}\left(u, u^{h}\right)=0$ if we would use the original Kružkov entropies (2.9), if $\tilde{u}$ is replaced by $u$, and if the exact time derivative and space gradient are used. As we prove in Proposition 3.2, this 
term is essentially bounded by a quantity proportional to $2 / M$, which is the size of the support of $\frac{d^{2} U}{d u^{2}}$.

Our treatment of the term $R_{\epsilon_{0}, \epsilon}^{h}\left(u, u^{h}\right)$ is different from [28] and [41], where the $L^{1}$ contraction property was used. Our motivation (for the application to formally high-order schemes) is to obtain a lower bound for $R_{\epsilon_{0}, \epsilon}^{h}\left(u, u^{h}\right)$ independent of the modulus of continuity of the function $u^{h}:[0, T] \rightarrow L^{1}\left(\mathbf{R}^{d}\right)$.

Lemma 3.1 (Lower bound for $R_{\epsilon_{0}, \epsilon}^{h}\left(u, u^{h}\right)$ ). We have

$$
\begin{aligned}
2 R_{\epsilon_{0}, \epsilon}^{h}( & \left.u, u^{h}\right) \\
\geq & \int_{\mathbf{R}^{d}} U_{M}\left(u^{h}\left(T, x^{\prime}\right), u\left(T, x^{\prime}\right)\right) d x^{\prime}-\int_{\mathbf{R}^{d}} U_{M}\left(u^{h}\left(0, x^{\prime}\right), u\left(0, x^{\prime}\right)\right) d x^{\prime} \\
& -2 \int_{Q_{T}^{\prime}} \omega_{\epsilon_{0}}\left(t^{\prime}\right) U_{M}\left(u^{h}\left(t^{\prime}, x^{\prime}\right), u\left(t^{\prime}, x^{\prime}\right)\right) d x^{\prime} d t^{\prime} \\
& -3\left(\epsilon+\epsilon_{0}\left\|\frac{d f}{d u}\left(u_{0}\right)\right\|_{L^{\infty}}\right) T V\left(u_{0}\right) .
\end{aligned}
$$

Proof. In view of (3.8b), we can write $R_{\epsilon_{0}, \epsilon}^{h}=R_{1}+R_{2}+R_{3}+R_{4}$, with the obvious notation. We estimate $R_{2}$ in the following crude manner: $R_{2} \geq 0$. In order to estimate $R_{1}$, we consider the decomposition

$$
\begin{aligned}
& U_{M}\left(u^{h}\left(T, x^{\prime}\right), u(t, x)\right) \\
&= U_{M}\left(u^{h}\left(T, x^{\prime}\right), u\left(T, x^{\prime}\right)\right) \\
&+\left\{U_{M}\left(u^{h}\left(T, x^{\prime}\right), u(T, x)\right)-U_{M}\left(u^{h}\left(T, x^{\prime}\right), u\left(T, x^{\prime}\right)\right)\right\} \\
&+\left\{U_{M}\left(u^{h}\left(T, x^{\prime}\right), u(t, x)\right)-U_{M}\left(u^{h}\left(T, x^{\prime}\right), u(T, x)\right)\right\},
\end{aligned}
$$

and get

$$
\begin{aligned}
& U_{M}\left(u^{h}\left(T, x^{\prime}\right), u(t, x)\right) \\
& \quad \geq U_{M}\left(u^{h}\left(T, x^{\prime}\right), u\left(T, x^{\prime}\right)\right)-\left|z i(T, x)-u\left(T, x^{\prime}\right)\right|-|u(t, x)-u(T, x)|,
\end{aligned}
$$

since the Lipschitz norm of $U_{M}$ is 1 . Taking into account the fact that $u$ is the entropy solution, as was done by Kuznetsov, we obtain

$$
\begin{aligned}
R_{1} \geq & \frac{1}{2} \int_{\mathbf{R}^{d}} U_{M}\left(u^{h}\left(T, x^{\prime}\right), u\left(T, x^{\prime}\right)\right) d x^{\prime} \\
& -\frac{1}{2} \epsilon T V\left(u_{0}\right)-\frac{1}{2} \epsilon_{0}\left\|\frac{d f}{d u}\left(u_{0}\right)\right\|_{L^{\infty}} T V\left(u_{0}\right) .
\end{aligned}
$$

We proceed in a similar way to estimate $R_{3}$ and $K_{4}$ :

$$
\begin{aligned}
R_{3} \geq & -\frac{1}{2} \int_{\mathbf{R}^{d}} U_{M}\left(u^{h}\left(0, x^{\prime}\right), u(0, x)\right) d x^{\prime}-\frac{1}{2}\left(\epsilon+\epsilon_{0}\left\|\frac{d f}{d u}\left(u_{0}\right)\right\|_{L^{\infty}}\right) T V\left(u_{0}\right), \\
R_{4} \geq & -\int_{Q_{T}^{\prime}} \omega_{\epsilon_{0}}\left(t^{\prime}\right) U_{M}\left(u^{h}\left(t^{\prime}, x^{\prime}\right), u\left(t^{\prime}, x^{\prime}\right)\right) d x^{\prime} d t^{\prime} \\
& -\frac{1}{2}\left(\epsilon+\epsilon_{0}\left\|\frac{d f}{d u}\left(u_{0}\right)\right\|_{L^{\infty}}\right) T V\left(u_{0}\right) .
\end{aligned}
$$


The desired result follows by adding the above inequalities.

For clarity in the following statement, we render here explicit the dependence of $S_{\epsilon_{0}, \epsilon}^{h}\left(u, u^{h}\right), E_{\epsilon_{0}, \epsilon}^{h}\left(u, u^{h}\right)$ and $E_{\epsilon_{0}, \epsilon}^{h}\left(u^{h}, u\right)$ upon $T$ by writing, instead, $S_{\epsilon_{0}, \epsilon}^{h}\left(u, u^{h} ; T\right), E_{\epsilon_{0}, \epsilon}^{h}\left(u, u^{h} ; T\right)$, and $E_{\epsilon_{0}, \epsilon}^{h}\left(u^{h}, u ; T\right)$, respectively. Our basic approximation result follows from Lemma 3.1 by an application of Gronwall's inequality.

Proposition 3.1 (Basic approximation inequality). We have

$$
\begin{aligned}
& \int_{\mathbf{R}^{d}}\left|u^{h}(T, x)-u(T, x)\right| d x \\
& \leq \frac{\left|\Omega^{h}(T)\right|}{2 M}+3 \int_{\mathbf{R}^{d}}\left|u^{h}(0, x)-u(0, x)\right| d x \\
&+9\left(\epsilon+\epsilon_{0}\left\|\frac{d f}{d u}\left(u_{0}\right)\right\|_{L^{\infty}}\right) T V\left(u_{0}\right) \\
&+6 \sup _{0 \leq t \leq T}\left(S_{\epsilon_{0}, \epsilon}^{h}\left(u, u^{h} ; t\right)+E_{\epsilon_{0}, \epsilon}^{h}\left(u, u^{h} ; t\right)+E_{\epsilon_{0}, \epsilon}^{h}\left(u^{h}, u ; t\right)\right),
\end{aligned}
$$

where $\Omega^{h}(T)$ is the union of the supports of $u$ and $u^{h}$.

Proof. By Lemma 3.1, we have

$$
\begin{aligned}
\int_{\mathbf{R}^{d}} & U_{M}\left(u^{h}\left(T, x^{\prime}\right), u\left(T, x^{\prime}\right)\right) d x^{\prime} \\
& \leq \mathbf{A}+2 \int_{Q_{T}^{\prime}} \omega_{\epsilon_{0}}\left(t^{\prime}\right) U_{M}\left(u^{h}\left(t^{\prime}, x^{\prime}\right), u\left(t^{\prime}, x^{\prime}\right)\right) d x^{\prime} d t^{\prime},
\end{aligned}
$$

where

$$
\begin{aligned}
\mathbf{A}= & \int_{\mathbf{R}^{d}} U_{M}\left(u^{h}\left(0, x^{\prime}\right), u\left(0, x^{\prime}\right)\right) d x^{\prime}+3\left(\epsilon+\epsilon_{0}\left\|\frac{d f}{d u}\left(u_{0}\right)\right\|_{L^{\infty}}\right) T V\left(u_{0}\right) \\
& +2 \sup _{0 \leq t \leq T}\left(S_{\epsilon_{0}, \epsilon}^{h}\left(u, u^{h} ; t\right)+E_{\epsilon_{0}, \epsilon}^{h}\left(u, u^{h} ; t\right)+E_{\epsilon_{0}, \epsilon}^{h}\left(u^{h}, u ; t\right)\right) .
\end{aligned}
$$

A simple application of Gronwall's inequality gives

$$
\int_{\mathbf{R}^{d}} U_{M}\left(u^{h}\left(T, x^{\prime}\right), u\left(T, x^{\prime}\right)\right) d x^{\prime} \leq \mathbf{A} \exp \left(2 \int_{0}^{T} \omega_{\epsilon_{0}}\left(t^{\prime}\right) d t^{\prime}\right),
$$

and the result follows from the fact that $\int_{0}^{T} \omega_{\epsilon_{0}}\left(t^{\prime}\right) d t^{\prime} \leq \frac{1}{2}$ (actually $\int_{0}^{T} \omega_{\epsilon_{0}}\left(t^{\prime}\right) d t^{\prime}$ $=\frac{1}{2}$ if $\left.T \geq \epsilon_{0}\right)$ and the inequalities, cf. (2.10),

$$
|w-v|-1 / 2 M \leq U_{M}(v, w) \leq|w-v| .
$$

3.2. Estimating the lack of symmetry of the entropy. In this subsection, we prove the following result.

Proposition 3.2 (Estimate of $\left.S_{\epsilon_{0}, \epsilon}^{h}\left(u, u^{h}\right)\right)$. We have

$S_{\epsilon_{0}, \epsilon}^{h}\left(u, u^{h}\right) \leq C T\left(\frac{h}{\epsilon}+\frac{\tau}{\epsilon_{0}}\left(1+C_{2} h^{-\beta}\right)+\frac{1}{M}\right) T V\left(u_{0}\right)+C \frac{h}{\epsilon}\left(\left\|u_{0}\right\|_{L^{\infty}}+C_{2} h^{-\beta}\right)$, where the constant $C$ does not depend upon $h, \epsilon, \epsilon_{0}, M, T$, and $u_{0}$. (The constant $C_{2}$ was introduced in (2.18).)

In view of the formula (3.8c), we can set $S_{\epsilon_{0}, \epsilon}^{h}\left(u, u^{h}\right)=S_{1}+S_{2}$ with obvious notation. The next two lemmas provide estimates for $S_{1}$ and $S_{2}$, respectively, which immediately imply the estimate stated in Proposition 3.2. 
Lemma 3.2 (Estimate of $S_{1}$ ). We have

$$
S_{1} \leq C T\left(\frac{\tau}{\epsilon_{0}}+\frac{1}{M}\right) T V\left(u_{0}\right)
$$

Lemma 3.3 (Estimate of $S_{2}$ ). We have

$$
S_{2} \leq C T\left(\frac{\tau}{\epsilon_{0}}+\frac{h}{\epsilon}\right) T V\left(u_{0}\right)+C \frac{h}{\epsilon}\left(\left\|u_{0}\right\|_{L^{\infty}}+C_{2} h^{-\beta}\right) .
$$

Proof of Lemma 3.2. We consider the decomposition $S_{1}=S_{1}^{\prime}+S_{1}^{\prime \prime}$, where

$$
2 S_{1}^{\prime}=-\int_{Q_{T}} \int_{Q_{T}^{\prime}}\left\{F_{M}\left(u, u^{h}\right)+F_{M}\left(u^{h}, u\right)\right\} \cdot \nabla \psi_{\epsilon}\left\{\tilde{\omega}_{\epsilon_{0}}-\tilde{\omega}_{\epsilon_{0}}^{\prime}\right\} d x d t d x^{\prime} d t^{\prime},
$$

and

$$
2 S_{1}^{\prime \prime}=\int_{Q_{T}} \int_{Q_{T}^{\prime}}\left\{F_{M}\left(u, u^{h}\right)-F_{M}\left(u^{h}, u\right)\right\} \cdot \nabla \psi_{\epsilon}\left\{\tilde{\omega}_{\epsilon_{0}}+\tilde{\omega}_{\epsilon_{0}}^{\prime}\right\} d x d t d x^{\prime} d t^{\prime} .
$$

We shall assume $u$ smooth. If $u$ is not smooth, it is not possible to integrate by parts as we do below. However, the formulas we shall derive are still valid, as is easily checked by a standard regularization argument. Using the divergence theorem, the definition (2.12) of $F_{M}$ and by (2.19), one gets

$$
\begin{aligned}
2\left|S_{1}^{\prime}\right| & \leq \int_{Q_{T}} \int_{Q_{T}^{\prime}}\left|\nabla \cdot\left\{F_{M}\left(u, u^{h}\right)+F_{M}\left(u^{h}, u\right)\right\}\right|\left|\tilde{\omega}_{\epsilon_{0}}-\tilde{\omega}_{\epsilon_{0}}^{\prime}\right| \psi_{\epsilon} d x d t d x^{\prime} d t^{\prime} \\
& \leq C \int_{Q_{T}}|\nabla \cdot u(t, x)|\left(\int_{0}^{T}\left|\tilde{\omega}_{\epsilon_{0}}-\tilde{\omega}_{\epsilon_{0}}^{\prime}\right| d t^{\prime}\right)\left(\int_{\mathbf{R}^{d}} \psi_{\epsilon} d x^{\prime}\right) d t d x \\
& \leq C \frac{\tau}{\epsilon_{0}} \int_{0}^{T} T V(u(t)) d t
\end{aligned}
$$

thus, since $T V(u(t))$ is a nonincreasing function of $t$,

$$
\left|S_{1}^{\prime}\right| \leq C T T V\left(u_{0}\right) \frac{\tau}{\epsilon_{0}} \text {. }
$$

Here we have used the following estimates:

$$
\begin{aligned}
& \int_{0}^{T}\left|\omega_{\epsilon_{0}}\left(t_{n+1}-t^{\prime}\right)-\omega_{\epsilon_{0}}\left(t-t^{\prime}\right)\right| d t^{\prime} \leq C \frac{\tau}{\epsilon_{0}} \quad \text { for } t \in\left[t_{n}, t_{n+1}\right), \\
& \int_{0}^{T}\left|\omega_{\epsilon_{0}}\left(t_{n+1}^{\prime}-t\right)-\omega_{\epsilon_{0}}\left(t-t^{\prime}\right)\right| d t^{\prime} \leq C \frac{\tau}{\epsilon_{0}} \quad \text { for } t \in\left[t_{n}^{\prime}, t_{n+1}^{\prime}\right) .
\end{aligned}
$$

On the other hand, by (2.13) and the assumption (2.19), we have

and thus,

$$
\left|\frac{\partial}{\partial u}\left(F_{M}(u, v)-F_{M}(v, u)\right)\right| \leq \frac{1}{2 M}\left\|\frac{d^{2} f}{d u^{2}}\right\|_{L^{\infty}} \leq \frac{C}{M},
$$

$$
\begin{aligned}
2\left|S_{1}^{\prime \prime}\right| & =\left|\int_{Q_{T}} \int_{Q_{T}^{\prime}}\left\{F_{M}\left(u, u^{h}\right)-F_{M}\left(u^{h}, u\right)\right\} \cdot \nabla \psi_{\epsilon}\left\{\tilde{\omega}_{\epsilon_{0}}+\tilde{\omega}_{\epsilon_{0}}^{\prime}\right\} d x d t d x^{\prime} d t^{\prime}\right| \\
& =\left|\int_{Q_{T}} \int_{Q_{T}^{\prime}} \nabla \cdot\left\{F_{M}\left(u, u^{h}\right)-F_{M}\left(u^{h}, u\right)\right\} \psi_{\epsilon}\left\{\tilde{\omega}_{\epsilon_{0}}+\tilde{\omega}_{\epsilon_{0}}^{\prime}\right\} d x^{\prime} d t^{\prime} d x d t\right| \\
& \leq \frac{C}{M} \int_{Q_{T}}|\nabla \cdot u(t, x)|\left(\int_{\mathbf{R}^{d}} \psi_{\epsilon} d x^{\prime}\right)\left(\int_{0}^{T}\left\{\tilde{\omega}_{\epsilon_{0}}+\tilde{\omega}_{\epsilon_{0}}^{\prime}\right\} d t^{\prime}\right) d x d t
\end{aligned}
$$


From the above inequality, we deduce that

$$
\left|S_{1}^{\prime \prime}\right| \leq C \frac{T}{M} T V\left(u_{0}\right)
$$

In view of (3.9) and (3.10), the proof of Lemma 3.2 is complete.

Proof of Lemma 3.3. As in the proof of Lemma 3.2, we can assume that $u$ is smooth. Consider the decomposition $S_{2}=S_{2}^{\prime}+S_{2}^{\prime \prime}$, where

$$
\begin{aligned}
& S_{2}^{\prime}=\int_{Q_{T}} \int_{Q^{\prime}{ }_{T}}\left\{U_{M}\left(\tilde{u}, u^{h}\right)-U_{M}\left(u, u^{h}\right)\right\} \tilde{\psi}_{\epsilon}^{\prime} \partial_{t} \omega_{\epsilon_{0}} d x d t d x^{\prime} d t^{\prime}, \\
& S_{2}^{\prime \prime}=-\int_{Q_{T}} \int_{Q^{\prime}{ }_{T}} U_{M}\left(u, u^{h}\right) \partial_{t} \omega_{\epsilon_{0}}\left\{\tilde{\psi}_{\epsilon}-\tilde{\psi}_{\epsilon}^{\prime}\right\} d x d t d x^{\prime} d t^{\prime} .
\end{aligned}
$$

In view of (3.2) and (3.3), we can estimate $S_{2}^{\prime}$ as follows:

$$
\begin{aligned}
\left|S_{2}^{\prime}\right| & \leq \int_{Q_{T}}|\tilde{u}(t, x)-u(t, x)|\left(\int_{\mathbf{R}^{d}} \tilde{\psi}_{\epsilon}^{\prime}\left(x ; x^{\prime}\right) d x^{\prime}\right)\left(\int_{0}^{T}\left|\partial_{t}^{\prime} \omega_{\epsilon_{0}}\right| d t^{\prime}\right) d x d t \\
& \leq T \sup _{0 \leq t \leq T}\|\tilde{u}(t)-u(t)\|_{L^{1}\left(\mathbf{R}^{d}\right)} \frac{C}{\epsilon_{0}} \sup _{K \in \mathscr{T}_{h}} \frac{1}{p_{K}} \sum_{e \in \partial K} \int_{e} \int_{\mathbf{R}^{d}} \psi_{\epsilon}\left(x-x^{\prime}\right) d x^{\prime} d \Gamma(x) .
\end{aligned}
$$

Since $u$ satisfies the $L^{1}$ contraction property, and in view of the definition (3.6) of $\tilde{u}$, one obtains

$$
\left|S_{2}^{\prime}\right| \leq C T \frac{\tau}{\epsilon_{0}} T V\left(u_{0}\right)
$$

To estimate $S_{2}^{\prime \prime}$, we integrate by parts, i.e.,

$$
\begin{aligned}
S_{2}^{\prime \prime}= & -\int_{Q_{T}} \int_{Q_{T}^{\prime}} \frac{\partial U_{M}}{d v}\left(u, u^{h}\right) \partial_{t} u \omega_{\epsilon_{0}}\left\{\tilde{\psi}_{\epsilon}-\tilde{\psi}_{\epsilon}^{\prime}\right\} d x d t d x^{\prime} d t^{\prime} \\
& +\int_{\mathbf{R}^{d}} \int_{Q_{T}^{\prime}} U_{M}\left(u(T, x), u^{h}\right) \omega_{\epsilon_{0}}\left(T-t^{\prime}\right)\left\{\tilde{\psi}_{\epsilon}-\tilde{\psi}_{\epsilon}^{\prime}\right\} d x d x^{\prime} d t^{\prime} \\
& -\int_{\mathbf{R}^{d}} \int_{Q_{T}^{\prime}} U_{M}\left(u(0, x), u^{h}\right) \omega_{\epsilon_{0}}\left(t^{\prime}\right)\left\{\tilde{\psi}_{\epsilon}-\tilde{\psi}_{\epsilon}^{\prime}\right\} d x d x^{\prime} d t^{\prime}
\end{aligned}
$$

In view of

$\int_{\mathbf{R}^{d}}\left|\tilde{\psi}_{\epsilon}\left(x ; x^{\prime}\right)-\psi_{\epsilon}\left(x-x^{\prime}\right)\right| d x^{\prime} \leq C \frac{h}{\epsilon}, \quad \int_{\mathbf{R}^{d}}\left|\tilde{\psi}_{\epsilon}^{\prime}\left(x ; x^{\prime}\right)-\psi_{\epsilon}\left(x-x^{\prime}\right)\right| d x^{\prime} \leq C \frac{h}{\epsilon}$, and (3.3), we get

$$
\begin{aligned}
& \left|\int_{Q_{T}} \int_{Q_{T}^{\prime}} \frac{\partial U_{M}}{\partial v}\left(u, u^{h}\right) \partial_{t} u \omega_{\epsilon_{0}}\left\{\tilde{\psi}_{\epsilon}-\tilde{\psi}_{\epsilon}^{\prime}\right\} d x d t d x^{\prime} d t^{\prime}\right| \\
& \quad \leq\left\|\frac{\partial U_{M}}{\partial v}\right\|_{L^{\infty}} \int_{Q_{T}}\left|\partial_{t} u(t, x)\right| \int_{\mathbf{R}^{d}}\left|\tilde{\psi}_{\epsilon}-\tilde{\psi}_{\epsilon}^{\prime}\right|\left(\int_{0}^{T} \omega_{\epsilon_{0}} d t^{\prime}\right) d x^{\prime} d t \dot{i} x \\
& \quad \leq C T \frac{h}{\epsilon} T V\left(u_{0}\right) .
\end{aligned}
$$


The remaining two terms are easily estimated; for instance, using (2.18), we have

$$
\begin{aligned}
& \left|\int_{\mathbf{R}^{d}} \int_{Q_{T}^{\prime}} U_{M}\left(u(T, x), u^{h}\right) \omega_{\epsilon_{0}}\left(T-t^{\prime}\right)\left\{\tilde{\psi}_{\epsilon}-\tilde{\psi}_{\epsilon}^{\prime}\right\} d x d x^{\prime} d t^{\prime}\right| \\
& \quad \leq C\left(\left\|u_{0}\right\|_{L^{\infty}\left(\mathbf{R}^{d}\right)}+\left\|u^{h}\right\|_{L^{\infty}\left([0, T] \times \mathbf{R}^{d}\right)}\right) \frac{h}{\epsilon} \\
& \quad \leq C\left(\left\|u_{0}\right\|_{L^{\infty}\left(\mathbf{R}^{d}\right)}+C_{2} h^{-\beta}\right) \frac{h}{\epsilon} .
\end{aligned}
$$

This shows that

$$
\left|S_{2}^{\prime}\right| \leq C \frac{h}{\epsilon}\left(\left\|u_{0}\right\|_{L^{\infty}\left(\mathbf{R}^{d}\right)}+C_{2} h^{-\beta}+T T V\left(u_{0}\right)\right) .
$$

The proof of Lemma 3.3 is complete in view of inequalities (3.11) and (3.12).

\subsection{Estimating the entropy production for the exact solution.}

Proposition 3.3 (Estimate of $E_{\epsilon_{0}, \epsilon}^{h}\left(u, u^{h}\right)$ ). We have

$$
E_{\epsilon_{0}, \epsilon}^{h}\left(u, u^{h}\right) \leq C \frac{h}{\epsilon}\left(\left\|u_{0}\right\|_{L^{\infty}\left(\mathbf{R}^{d}\right)}+C_{2} h^{-\beta}+T T V\left(u_{0}\right)\right)
$$

where the constant $C$ does not depend upon $h, \epsilon, \epsilon_{0}, M, T$, and $u_{0}$. (The constant $C_{2}$ was introduced in (2.18).)

Proof. Since $u$ is an entropy solution to (1.1), for each $\left(t^{\prime}, x^{\prime}\right)$ and $n$, one has

$$
\begin{gathered}
\int_{\mathbf{R}^{d}}\left\{U_{M}\left(u\left(t_{n+1}, x\right), u^{h}\left(t^{\prime}, x^{\prime}\right)\right)-U_{M}\left(u\left(t_{n}, x\right), u^{h}\left(t^{\prime}, x^{\prime}\right)\right)\right\} \psi_{\epsilon}\left(x-x^{\prime}\right) d x \\
-\int_{t_{n}}^{t_{n+1}} \int_{\mathbf{R}^{d}} F_{M}\left(u(t, x), u^{h}\right) \nabla \cdot \psi_{\epsilon}\left(x-x^{\prime}\right) d t d x \leq 0 .
\end{gathered}
$$

Multiplying this inequality by $\omega_{\epsilon_{0}}\left(t_{n+1}-t^{\prime}\right)$, using (3.2), and summing in time, we arrive at the following inequality:

$$
\begin{aligned}
& -\sum_{n=0}^{n_{T}-1} \int_{\mathbf{R}^{d}} U_{M}\left(u\left(t_{n}, x\right), u^{h}\right)\left\{\omega_{\epsilon_{0}}\left(t_{n+1}-t^{\prime}\right)-\omega_{\epsilon_{0}}\left(t_{n}-t^{\prime}\right)\right\} \psi_{\epsilon}\left(x-x^{\prime}\right) d x \\
& \quad-\int_{Q_{T}} F_{M}\left(u(t, x), u^{h}\right) \nabla \psi_{\epsilon}\left(x-x^{\prime}\right) \tilde{\omega}_{\epsilon_{0}}\left(t ; t^{\prime}\right) d t d x \\
& \quad+\int_{\mathbf{R}^{d}} U_{M}\left(u(T, x), u^{h}\right) \omega_{\epsilon_{0}}\left(T-t^{\prime}\right) \psi_{\epsilon}\left(x-x^{\prime}\right) d x \\
& \quad-\int_{\mathbf{R}^{d}} U_{M}\left(u(0, x), u^{h}\right) \omega_{\epsilon_{0}}\left(t^{\prime}\right) \psi_{\epsilon}\left(x-x^{\prime}\right) d x \leq 0 .
\end{aligned}
$$

By the definition of $\tilde{u},(3.6)$, we have the identity

$$
\begin{gathered}
\int_{\mathbf{R}^{d}} U_{M}\left(u\left(t_{n}, x\right), u^{h}\right)\left\{\omega_{\epsilon_{0}}\left(t_{n+1}-t^{\prime}\right)-\omega_{\epsilon_{0}}\left(t_{n}-t^{\prime}\right)\right\} \psi_{\epsilon}\left(x-x^{\prime}\right) d x \\
=\int_{t_{n}}^{t_{n+1}} \int_{\mathbf{R}^{d}} U_{M}\left(\tilde{u}(t, x), u^{h}\right) \partial_{t} \omega_{\epsilon_{0}}\left(t-t^{\prime}\right) \psi_{\epsilon}\left(x-x^{\prime}\right) d x d t,
\end{gathered}
$$


so $E_{\epsilon_{0}, \epsilon}^{h}\left(u, u^{h}\right)$ defined by (3.7) is bounded above by the quantity

$$
\int_{Q_{r}} \int_{Q_{T}^{\prime}} U_{M}\left(\tilde{u}(t, x), u^{h}\right) \partial_{t} \omega_{\epsilon_{0}}\left(t-t^{\prime}\right)\left\{\psi_{\epsilon}\left(x-x^{\prime}\right)-\tilde{\psi}_{\epsilon}\left(x ; x^{\prime}\right)\right\} d t d x d t^{\prime} d x^{\prime} .
$$

Using integration by parts in time, we can rewrite this term as follows:

$$
\begin{aligned}
\int_{Q_{T} \times Q_{T}^{\prime}} & U_{M}\left(\tilde{u}(t, x), u^{h}\right) \partial_{t} \omega_{\epsilon_{0}}\left(t-t^{\prime}\right)\left\{\psi_{\epsilon}\left(x-x^{\prime}\right)-\tilde{\psi}_{\epsilon}\left(x ; x^{\prime}\right)\right\} d t d x d t^{\prime} d x^{\prime} \\
= & -\sum_{n=0}^{n_{T}-1} \int_{\mathbf{R}^{d} \times Q_{T}^{\prime}}\left\{U_{M}\left(u\left(t_{n+1}, x\right), u^{h}\right)-U_{M}\left(u\left(t_{n}, x\right), u^{h}\right)\right\} \\
& \left.+\int_{\mathbf{R}^{d}} \int_{Q_{T}^{\prime}} U_{M}\left(u(T, x), u_{\epsilon}-\tilde{\psi}_{\epsilon}\right) \omega_{\epsilon_{0}}\left(t_{n+1}-t^{\prime}\right) d x d x_{\epsilon}\left(x-x^{\prime}\right)-\tilde{\psi}_{\epsilon}\left(x ; x^{\prime}\right)\right\} \omega_{\epsilon_{0}}\left(T-t^{\prime}\right) d x d x^{\prime} d t^{\prime} \\
& -\int_{\mathbf{R}^{d}} \int_{Q_{T}^{\prime}} U_{M}\left(u(0, x), u^{h}\right)\left\{\psi_{\epsilon}\left(x-x^{\prime}\right)-\tilde{\psi}_{\epsilon}\left(x ; x^{\prime}\right)\right\} \omega_{\epsilon_{0}}\left(t^{\prime}\right) d x d x^{\prime} d t^{\prime} .
\end{aligned}
$$

Each term in the right-hand side above can be estimated along the lines in the proof of Lemma 3.3. We omit the details.

3.4. Estimating the entropy production for the approximate solution. In this subsection, we prove the following result.

Proposition 3.4 (Estimate of $E_{\epsilon_{0}, \epsilon}^{h}\left(u, u^{h}\right)$ ). We have

$$
\begin{aligned}
E_{\epsilon_{0}, \epsilon}^{h}\left(u^{h}, u\right) \leq C & \frac{h}{\epsilon}\left(\left\|u_{0}\right\|_{L^{\infty}}+C_{2} h^{-\beta}\right)+M C_{1} h^{\alpha} \\
& +C\left(T\left|\Omega^{h}(T)\right|\right)^{1 / 2} \frac{h^{\frac{1}{2}}}{\epsilon}\left(\left\|u_{0}\right\|_{L^{2}\left(\mathbf{R}^{d}\right)}+C_{1}^{1 / 2} h^{\alpha / 2}\right),
\end{aligned}
$$

where the constant $C$ does not depend upon $h, \epsilon, \epsilon_{0}, M, T$, and $u_{0}$.

This result is a direct consequence of Lemmas 3.4, 3.5, 3.6, and 3.7, which we prove in the remainder of this section.

Lemma 3.4. We have

$$
E_{\epsilon_{0}, \epsilon}^{h}\left(u^{h}, u\right) \leq E_{1}+E_{2},
$$

where

$$
\begin{aligned}
E_{1}= & \int_{Q_{T}} \sum_{n=0}^{n_{T}-1} \sum_{\substack{k \in \mathcal{F}_{h} \\
e \in \mathcal{K}_{K}}} \frac{|e||K|}{p_{K}} U_{M}\left(u_{K, e}^{n+1}, u\right)\left(\tilde{\psi}_{\epsilon}^{\prime}(x)-\tilde{\psi}_{\epsilon, e}^{\prime}(x)\right) \omega_{\epsilon_{0}}\left(t_{n+1}^{\prime}-t\right) d x d t \\
& +\int_{Q_{T}} \int_{\mathbf{R}^{d}} U_{M}\left(u^{h}\left(T, x^{\prime}\right), u\right) \omega_{\epsilon_{0}}(T-t)\left\{\psi_{\epsilon}\left(x-x^{\prime}\right)-\tilde{\psi}_{\epsilon}^{\prime}\left(x ; x^{\prime}\right)\right\} d t d x d x^{\prime} \\
& -\int_{Q_{T}} \int_{\mathbf{R}^{d}} U_{M}\left(u^{h}\left(0, x^{\prime}\right), u\right) \omega_{\epsilon_{0}}(T)\left\{\psi_{\epsilon}\left(x-x^{\prime}\right)-\tilde{\psi}_{\epsilon}^{\prime}\left(x ; x^{\prime}\right)\right\} d t d x d x^{\prime},
\end{aligned}
$$

and

$$
\begin{aligned}
E_{2}=\int_{Q_{T}} \sum_{n=0}^{n_{T}-1} \sum_{\substack{K \in \mathcal{F}_{h} \\
e \in \partial K}} a_{K, e}^{n}\left\{\frac{\partial U_{M}}{\partial v}\left(v_{K, e}^{n}, u\right)-\right. & \left.\frac{\partial U_{M}}{\partial v}\left(v_{K_{e}, e}^{n}, u\right)\right\} \\
& \cdot \tilde{\psi}_{\epsilon, e}^{\prime}(x) \omega_{\epsilon_{0}}\left(t_{n+1}^{\prime}-t\right) \tau|e| d t d x .
\end{aligned}
$$


Note that, if we set

$$
\tilde{\psi}_{\epsilon, e^{\prime}}^{\prime}(x)=\frac{1}{\left|e^{\prime}\right|} \int_{e^{\prime}} \psi_{\epsilon}\left(x-y^{\prime}\right) d \Gamma\left(y^{\prime}\right), \quad e^{\prime} \in \partial K^{\prime}, \quad K^{\prime} \in \mathscr{T}_{h},
$$

then, in view of (3.3), we have

$$
\tilde{\psi}_{\epsilon}^{\prime}\left(x ; x^{\prime}\right)=\frac{1}{p_{K}^{\prime}} \sum_{e^{\prime} \in \partial K^{\prime}} \tilde{\psi}_{\epsilon, e^{\prime}}^{\prime}(x)\left|e^{\prime}\right|, \quad x \in \mathbf{R}^{d}, x^{\prime} \in K^{\prime}, \quad K^{\prime} \in \mathscr{T}_{h},
$$

by the definition of $\tilde{\psi}_{\epsilon}^{\prime}$; cf. (3.4). We use this identity in the proof below.

Proof of Lemma 3.4. Following [9], we use the entropy inequalities (2.15) as follows: we sum the entropy inequalities $(2.15)$, written for the polyhedron $K$ and for the polyhedron $K_{e}$, respectively; in view of the conservation properties, (2.14a) and (2.17), the numerical entropy fluxes cancel each other, and only the exact entropy fluxes remain; then we get

$$
\begin{aligned}
& \frac{|K|}{p_{K}} U_{M}\left(u_{K, e}^{n+1}, c\right)+\frac{\left|K_{e}\right|}{p_{K_{e}}} U_{M}\left(u_{K_{e}, e}^{n+1}, c\right)+\tau\left\{F_{M}\left(v_{K_{e}}^{n}, c\right)-F_{M}\left(u_{K}^{n}, c\right)\right\} \cdot N_{K, e} \\
& \quad \leq \tau a_{K, e}^{n}\left\{\frac{|K|}{p_{K}} \frac{\partial U_{M}}{\partial v}\left(v_{K}^{n}, c\right)-\frac{\left|K_{e}\right|}{p_{K_{e}}} \frac{\partial U_{M}}{\partial v}\left(v_{K_{e}}^{n}, c\right)\right\} .
\end{aligned}
$$

We multiply the last inequality by $\tilde{\psi}_{\epsilon, e}^{\prime}|e|$ and sum over all faces and all polyhedra:

$$
\begin{aligned}
\sum_{K \in \mathscr{T}_{h}} & |K| \sum_{e \in \partial K} \frac{|e|}{p_{K}} U_{M}\left(u_{K, e}^{n+1}, c\right) \psi_{\epsilon, e}^{h}-\sum_{K \in \mathscr{T}_{h}} U_{M}\left(u_{K, e}^{n+1}, c\right) \psi_{\epsilon}^{h}|K| \\
& +\sum_{\substack{K \in \mathscr{F}_{h} \\
e \in \partial K}} \int_{e} F_{M}\left(u_{K}^{n}, c\right) \cdot N_{K, e} \psi_{\epsilon}\left(x-x^{\prime}\right) d \Gamma\left(x^{\prime}\right) \\
\leq & \sum_{K \in \mathscr{T}_{h}} \sum_{e \in \partial K} a_{K, e}^{n}\left\{\frac{\partial U_{M}}{\partial v}\left(v_{K, e}^{n+1}, c\right)-\frac{\partial U_{M}}{\partial v}\left(v_{K_{e}, e}^{n+1}, c\right)\right\} \tilde{\psi}_{\epsilon, e}^{\prime} \tau|e| .
\end{aligned}
$$

Observe that for each polyhedron $K$

$$
\sum_{e \in \partial K} \int_{e} F_{M}\left(u_{K}^{n}, c\right) \cdot N_{K, e} \psi_{\epsilon}\left(x-x^{\prime}\right) d \Gamma\left(x^{\prime}\right)=-\int_{K} F_{M}\left(u_{K}^{n}, c\right) \cdot \nabla \psi_{\epsilon}\left(x-x^{\prime}\right) d x^{\prime} .
$$

We multiply the last inequality by $\omega_{\epsilon_{0}}\left(t_{n+1}^{\prime}-t\right)$ and sum with respect to the time variable:

$$
\begin{aligned}
& -\int_{Q_{T}^{\prime}} U_{M}\left(u^{h}\left(t^{\prime}, x^{\prime}\right), c\right) \tilde{\psi}_{\epsilon}^{\prime} \partial_{t^{\prime}} \omega_{\epsilon_{0}}+F_{M}\left(u^{h}, c\right) \cdot \nabla^{\prime} \psi_{\epsilon} \tilde{\omega}_{\epsilon_{0}}^{\prime}\left(t ; t^{\prime}\right) d t^{\prime} d x^{\prime} \\
& +\int_{\mathbf{R}^{d}} U_{M}\left(u^{h}\left(T, x^{\prime}\right), c\right) \tilde{\psi}_{\epsilon}^{\prime} \omega_{\epsilon_{0}}(T-t) d x^{\prime}-\int_{\mathbf{R}^{d}} U_{M}\left(u^{h}\left(0, x^{\prime}\right), c\right) \tilde{\psi}_{\epsilon}^{\prime} \omega_{\epsilon_{0}}(t) d x^{\prime} \\
& \quad \leq \sum_{n=0}^{n_{T}-1} \sum_{K \in \mathscr{T}_{h}}|K| \omega_{\epsilon_{0}}\left(t_{n+1}^{\prime}-t\right)\left\{U_{M}\left(u_{K}^{n+1}, c\right) \tilde{\psi}_{\epsilon}^{\prime}-\frac{1}{p_{K}} \sum_{e \in \partial K} U_{M}\left(u_{K, e}^{n+1}, c\right) \tilde{\psi}_{\epsilon, e}^{\prime}|e|\right\} \\
& \quad+\sum_{n=0}^{n_{T}^{\prime}-1} \sum_{\substack{K \in \mathscr{F}_{h} \\
e \in \partial K}} a_{K, e}^{n}\left\{\frac{\partial U_{M}}{\partial v}\left(v_{K, e}^{n+1}, c\right)-\frac{\partial U_{M}}{\partial v}\left(v_{K_{e}, e}^{n+1}, c\right)\right\} \tilde{\psi}_{\epsilon, e}^{\prime} \omega_{\epsilon_{0}}\left(t_{n+1}^{\prime}-t\right) \tau|e| .
\end{aligned}
$$


The statement in Lemma 3.4 then is a consequence of Jensen's inequality applied to the convex decomposition (2.8).

The following a priori estimate of the entropy dissipation of the scheme was derived in [9].

Lemma 3.5. We have

$$
\sum_{n=0}^{n_{T}-1} \sum_{\substack{K \in \mathcal{F}_{h} \\ e \in \partial K}} \frac{|e||K|}{p_{K}}\left(u_{K, e}^{n+1}-u_{K}^{n+1}\right)^{2} \leq\left\|u_{0}\right\|_{L^{2}\left(\mathbf{R}^{d}\right)}^{2}+C_{1} h^{\alpha},
$$

where $C_{1}$ was introduced in (2.16).

Proof. The entropy inequalities (2.15) hold for all $M$, so, for each $h$ fixed, we can take $M$ to be larger than $\left\|u^{h}\right\|_{L^{\infty}}$, and in view of (2.10), condition (2.15) then reduces to a discrete entropy inequality for the quadratic entropy $u^{2}$. By summation in space and time, we can deduce from this quadratic entropy inequality that

$$
\begin{aligned}
& \sum_{K \in \mathscr{T}_{h}}\left(u_{K}^{n_{T}}\right)^{2}|K|+\sum_{n=0}^{n_{T}-1} \sum_{\substack{\boldsymbol{c} \in \mathcal{O}_{h} \\
e \in \partial K}} \frac{|e||K|}{p_{K}}\left(v_{K, e}^{n+1}-v_{K}^{n+1}\right)^{2} \\
& \quad \leq \sum_{K \in \mathscr{T}_{h}}\left(u_{K}^{0}\right)^{2}|K|+\sum_{n=0}^{n_{T}-1} \sum_{\substack{K \in \mathcal{F}_{h} \\
e \in \partial K}}\left|a_{K, e}^{n} \| u_{K_{e}}^{n+1}-u_{K_{e}, e}^{n+1}\right||e| \tau .
\end{aligned}
$$

The proof is based on the arguments already used in the proof of Lemma 3.4. See [9] for a proof. The proof is complete in view of (2.10).

In the derivation of (3.14), the assumption (2.16) is used to estimate the entropy production only. When the antidiffusive term $a_{K, e}^{n}$ vanishes identically, (3.14) holds with $C_{1}=0$.

Equipped with (3.14), we now turn to estimating $E_{1}$ and $E_{2}$. In view of Lemma 3.4, Proposition 3.4 is an immediate consequence of Lemmas 3.6 and 3.7 below.

Lemma 3.6 (Estimate of $E_{1}$ ). We have

$$
E_{1} \leq C \frac{h}{\epsilon}\left(\left\|u_{0}\right\|_{L^{\infty}}+C_{2} h^{-\beta}\right)+C\left(T\left|\Omega^{h}(T)\right|\right)^{1 / 2} \frac{h^{\frac{1}{2}}}{\epsilon}\left(\left\|u_{0}\right\|_{L^{2}\left(\mathbf{R}^{d}\right)}+C_{1}^{1 / 2} h^{\alpha / 2}\right) .
$$

Proof. In view of the identity (3.10b), for all $K \in \mathscr{T}_{h}$ we have

$$
\frac{1}{p_{K}} \sum_{e \in \partial K} U_{M}\left(u_{K}^{n+1}, u\right)\left\{\tilde{\psi}_{\epsilon}-\tilde{\psi}_{\epsilon, e}^{h}\right\}|e|=0,
$$

and thus $E_{1}$ can be rewritten in the form

$$
E_{1}=\int_{Q_{T}}\left\{\theta_{1}(t, x)+\theta_{2}(t, x)+\theta_{3}(t, x)\right\} d t d x,
$$


with

$$
\begin{aligned}
& \theta_{1}(t, x)=\sum_{n=0}^{n_{T}} \sum_{\substack{K \in \mathcal{F}_{h} \\
e \in \partial K}} \frac{|e \| K|}{p_{K}}\left\{U_{M}\left(u_{K, e}^{n+1}, u\right)-U_{M}\left(u_{K}^{n+1}, u\right)\right\} \\
& \cdot\left(\tilde{\psi}_{\epsilon}^{\prime}-\tilde{\psi}_{\epsilon, e}^{\prime}\right) \omega_{\epsilon_{0}}\left(t_{n+1}^{\prime}-t\right), \\
& \theta_{2}(t, x)=\int_{\mathbf{R}^{d}} U_{M}\left(u^{h}\left(T, x^{\prime}\right), u\right) \omega_{\epsilon_{0}}(T-t)\left\{\psi_{\epsilon}\left(x-x^{\prime}\right)-\tilde{\psi}_{\epsilon}^{\prime}\left(x ; x^{\prime}\right)\right\} d x^{\prime}, \\
& \theta_{3}(t, x)=-\int_{\mathbf{R}^{d}} U_{M}\left(u^{h}\left(0, x^{\prime}\right), u\right) \omega_{\epsilon_{0}}(t)\left\{\psi_{\epsilon}\left(x-x^{\prime}\right)-\tilde{\psi}_{\epsilon}^{\prime}\left(x ; x^{\prime}\right)\right\} d x^{\prime} .
\end{aligned}
$$

In order to estimate $\theta_{1}$, we write

$$
\begin{aligned}
\left|\int_{Q_{T}} \theta_{1}(t, x) d x d t\right| & \leq \sum_{n=0}^{n_{T}-1} \sum_{\substack{K \in \mathscr{Y}_{h} \\
e \in \partial K}} \frac{|e||K|}{p_{K}}\left|u_{K, e}^{n+\mathrm{i}}-u_{K}^{n+1}\right| \int_{\mathbf{R}^{d}}\left|\tilde{\psi}_{\epsilon}^{\prime}-\tilde{\psi}_{\epsilon, e}^{\prime}\right| d x \\
& \leq \frac{C}{\epsilon} \sum_{n=0}^{n_{T}-1} \sum_{\substack{K \in \mathcal{F}_{h} \\
e \in \partial K}}\left|u_{K, e}^{n+1}-u_{K}^{n+1}\right| \frac{|K||e|}{p_{K}} h,
\end{aligned}
$$

where we have used the following estimate valid for all $x^{\prime}$ :

$$
\int_{\mathbf{R}^{d}}\left|\tilde{\psi}_{\epsilon}^{\prime}\left(x ; x^{\prime}\right)-\tilde{\psi}_{\epsilon, e}^{\prime}\left(x ; x^{\prime}\right)\right| d x \leq C \frac{h}{\epsilon} .
$$

Since the triangulation satisfies condition (2.1), and by using the CauchySchwarz inequality, we deduce that

$$
\begin{aligned}
\left|\int_{Q_{T}} \theta_{1}(t, x) d t d x\right| & \leq C \frac{\gamma}{\epsilon} \sum_{n=0}^{n_{T}-1} \sum_{\substack{K \in \mathscr{F}_{h} \\
e \in \partial K}}\left|u_{K, e}^{n+1}-u_{K}^{n+1}\right| \frac{|K||e|}{p_{K}} \tau \\
& \leq C \frac{\gamma}{\epsilon}\left(T\left|\Omega^{h}(T)\right|\right)^{1 / 2}\left(\sum_{\substack{n=0 \\
n_{T}-1}} \sum_{\substack{K \in \mathcal{Y}_{h} \\
e \in \partial K}}\left|u_{K, e}^{n+1}-u_{K}^{n+1}\right|^{2} \frac{|K||e|}{p_{K}} \tau\right)^{1 / 2},
\end{aligned}
$$

where $\left|\Omega^{h}(T)\right|$ represents the Lebesgue measure of the support of the approximate solution at time $T$. Using the entropy dissipation estimate (3.13), we thus get

$$
\left|\int_{Q_{T}} \theta_{1}(t, x) d t d x\right| \leq C\left(T\left|\Omega^{h}(T)\right|\right)^{1 / 2} \frac{h^{\frac{1}{2}}}{\epsilon}\left(\left\|u_{0}\right\|_{L^{2}\left(\mathbf{R}^{d}\right)}+C_{1}^{1 / 2} h^{\alpha / 2}\right) .
$$

Similar arguments as those used in the proof of Lemma 3.3 yield the following estimate for $\theta_{2}$ and $\theta_{3}$ :

$$
\begin{aligned}
\int_{Q_{T}}\left\{\left|\theta_{2}(t, x)\right|+\left|\theta_{3}(t, x)\right|\right\} d t d x & \leq C \frac{h}{\epsilon}\left(\left\|u^{h}\right\|_{L^{\infty}\left([0, T) \times \mathbf{R}^{d}\right)}+\left\|u_{0}\right\|_{L^{\infty}}\right) \\
& \leq C \frac{h}{\epsilon}\left(\left\|u_{0}\right\|_{L^{\infty}}+C_{2} h^{-\beta}\right) .
\end{aligned}
$$

The proof of Lemma 3.6 is complete in view of (3.15) and (3.16).

Lemma 3.7 (Estimate of $E_{2}$ ). We have

$$
E_{2} \leq M C_{1} h^{\alpha} \text {. }
$$


Proof. By the definition of $E_{2}$ in Lemma 3.4, the definition (2.10) of $U_{M}$, and the property (2.11), one has

$$
\begin{aligned}
\left|E_{2}\right| & \leq M \sum_{n=0}^{n_{T}-1} \sum_{\substack{K \in \mathcal{F}_{h} \\
e \in \partial K}}\left|a_{K, e}^{n} \| v_{K, e}^{n+1}-v_{K_{e}, e}^{n+1}\right|\left(\int_{\mathbf{R}^{d}} \tilde{\psi}_{\epsilon, e}^{\prime}\left(x^{\prime} ; x\right) d x\right)\left(\int_{0}^{T} \omega_{\epsilon_{0}} d t\right) \tau|e| \\
& \leq M \sum_{n=0}^{n_{T}-1} \sum_{\substack{K \in \mathcal{F}_{h} \\
e \in \mathcal{F}_{K}}}\left|a_{K, e}^{n} \| v_{K, e}^{n+1}-v_{K_{e}, e}^{n+1}\right| \tau|e|,
\end{aligned}
$$

where we have used the following identity:

$$
\int_{\mathbf{R}^{d}} \tilde{\psi}_{\epsilon, e}(x) d x=\frac{1}{|e|} \int_{e}\left(\int_{\mathbf{R}^{d}} \tilde{\psi}_{\epsilon}\left(x ; x^{\prime}\right) d x\right) d \Gamma\left(x^{\prime}\right)=1 .
$$

The conclusion is a consequence of assumption (2.16).

3.5. Completion of the proof of Theorem 2.1. From Propositions 3.1 to 3.4, we immediately deduce that

$$
\left\|u^{h}(T)-u(T)\right\|_{L^{1}\left(\mathbf{R}^{d}\right)} \leq \mathbf{A}_{-1} M^{-1}+\mathbf{A}_{0}+\mathbf{A}_{1} M
$$

where

$$
\begin{aligned}
\mathbf{A}_{-1}= & C+C T T V\left(u_{0}\right), \\
\mathbf{A}_{0}= & 2\left\|\mathbf{P}_{h}\left(u_{0}\right)-u_{0}\right\|_{L^{1}\left(\mathbf{R}^{d}\right)}+C T V\left(u_{0}\right) \epsilon \\
& +\frac{C}{\epsilon}\left(\left(T T V\left(u_{0}\right)+\left\|u_{0}\right\|_{L^{\infty}\left(\mathbf{R}^{d}\right)}\right) h\right. \\
& \quad+T^{1 / 2}\left\|u_{0}\right\|_{L^{2}\left(\mathbb{R}^{d}\right)} h^{1 / 2}+T^{1 / 2} C_{1}^{1 / 2} h^{(1+\alpha) / 2} \\
& \left.\quad+\left(1+T T V\left(u_{0}\right)\right) C_{2} h^{1-\beta}\right), \\
\mathbf{A}_{1}= & C_{1} h^{\alpha},
\end{aligned}
$$

where $C$ depends on $\left|\Omega^{h}(T)\right|, \sigma, \gamma$, and on the ratio $\epsilon_{0}\left\|\frac{d f}{d u}\left(u_{0}\right)\right\|_{L^{\infty}} / \epsilon$, which we set equal to a fixed constant.

Minimizing over $M$, we obtain

$$
\left\|u^{h}(T)-u(T)\right\|_{L^{1}\left(\mathbf{R}^{d}\right)} \leq 2 \sqrt{\mathbf{A}_{-1} \mathbf{A}_{1}}+\mathbf{A}_{0},
$$

that is,

$$
\begin{aligned}
\left\|u^{h}(T)-u(T)\right\|_{L^{1}\left(\mathbf{R}^{d}\right)} \leq & 2\left\|\mathbf{P}_{h}\left(u_{0}\right)-u_{0}\right\|_{L^{1}\left(\mathbf{R}^{d}\right)}+C T V\left(u_{0}\right) \epsilon \\
& +\frac{C}{\epsilon}\left(\left(T T V\left(u_{0}\right)+\left\|u_{0}\right\|_{L^{\infty}\left(\mathbf{R}^{d}\right)}\right) h\right. \\
& \quad+T^{1 / 2}\left\|u_{0}\right\|_{L^{2}\left(\mathbf{R}^{d}\right)} h^{1 / 2}+T^{1 / 2} C_{1}^{1 / 2} h^{(1+\alpha) / 2} \\
& \left.+\left(1+T T V\left(u_{0}\right)\right) C_{2} h^{1-\beta}\right) \\
& +C C_{1}^{1 / 2} h^{\alpha / 2} .
\end{aligned}
$$


Minimizing over $\epsilon$, we get

$$
\begin{aligned}
& \left\|u^{h}(T)-u(T)\right\|_{L^{1}\left(\mathbf{R}^{d}\right)} \\
& \leq 2\left\|\mathbf{P}_{h}\left(u_{0}\right)-u_{0}\right\|_{L^{1}\left(\mathbf{R}^{d}\right)} \\
& +C T V\left(u_{0}\right)^{1 / 2}\left(\left(T^{1 / 2} T V\left(u_{0}\right)^{1 / 2}+\left\|u_{0}\right\|_{L^{\infty}\left(\mathbf{R}^{d}\right)}^{1 / 2}\right) h^{1 / 2}\right. \\
& +T^{1 / 4}\left\|u_{0}\right\|_{L^{2}\left(\mathbb{R}^{d}\right)}^{1 / 2} h^{1 / 4}+T^{1 / 4} C_{1}^{1 / 4} h^{1 / 4+\alpha / 4} \\
& \left.+\left(1+T^{1 / 2} T V\left(u_{0}\right)^{1 / 2}\right) C_{2}^{1 / 2} h^{1 / 2-\beta / 2}\right) \\
& +C C_{1}^{1 / 2} h^{\alpha / 2} .
\end{aligned}
$$

The result follows from the fact that $\left\|\mathbf{P}_{h}\left(u_{0}\right)-u_{0}\right\|_{L^{1}\left(\mathbf{R}^{d}\right)} \leq C h T V\left(u_{0}\right)$. (See, for example, [8] for a proof.) This completes the proof of Theorem 2.1 .

\section{THE ANTIDIFFUSION SCHEMES}

In this section, we prove that Theorem 2.1 does apply to the so-called modified antidiffusive flux schemes constructed from monotone schemes. For simplicity, we assume here that the underlying monotone scheme satisfies sharp entropy inequalities, in the sense of [14]. The antidiffusion schemes are formally high-order accurate schemes of the form (2.6) with a numerical flux $g_{e, K}^{n}$ given by

$$
g_{e, K}^{n}=g_{e, K}^{m n, n}+a_{e, K}^{n},
$$

where

$$
g_{e, K}^{m n, n} \text { is a two-point monotone flux, }
$$

and $a_{e, K}^{n}$ satisfies the conservativity property (2.17) and the estimate

$$
\left|a_{e, K}^{n}\right| \leq A h_{K}^{\gamma},
$$

for some number $\gamma$ in $(0,1]$. To apply Theorem 2.1 to the schemes under consideration, we only have to verify that the discrete entropy inequality (2.15), the estimate (2.16), and the $L^{\infty}$ bound (2.18) hold.

We first show that the entropy inequality (2.15) is satisfied. By $(2.8)$, we have

$$
u_{K}^{n+1}=\frac{1}{p_{K}} \sum_{e \in \partial K} u_{K, e}^{n+1}|e|
$$

where

$$
u_{K, e}^{n+1}=u_{K}^{n}-\frac{\tau p_{K}}{|K|}\left\{g_{e, K}^{m n, n}+a_{e, K}^{n}-N_{e, K} \cdot f\left(u_{K}^{n}\right)\right\}
$$

Setting

$$
w_{K, e}^{n+1}=u_{K, e}^{n+1}+\frac{\tau p_{K}}{|K|} a_{e, K}^{n}
$$

we get

$$
w_{K, e}^{n+1}=u_{K}^{n}-\frac{\tau p_{K}}{|K|}\left\{g_{e, K}^{m n, n}-N_{e, K} \cdot f\left(u_{K}^{n}\right)\right\}
$$


and so, by (4.1b) and the CFL condition (2.7),

$$
\begin{aligned}
U_{M}( & \left.\left.u_{K, e}^{n+1}, c\right)-U_{M}\left(u_{K}^{n}, c\right)+\frac{\tau p_{K}}{|K|}\left\{G_{e, K}^{n}-N_{e, K} \cdot F_{M}\left(u_{K}^{n}, c\right)\right)\right\} \\
& \leq U_{M}\left(u_{K, e}^{n+1}, c\right)-U_{M}\left(w_{K, e}^{n+1}, c\right) \\
& =\left(u_{K, e}^{n+1}-w_{K, e}^{n+1}\right) \frac{\partial U_{M}}{\partial v}\left(v_{K, e}^{n}, c\right)=-\frac{\tau p_{K}}{|K|} a_{K, e}^{n} \frac{\partial U_{M}}{\partial v}\left(v_{K, e}^{n}, c\right),
\end{aligned}
$$

where

$$
v_{K, e}^{n}=\left(1-\theta_{K, e}^{n}\right) u_{K, e}^{n+1}+\theta_{K, e}^{n} w_{K, e}^{n+1}=u_{K, e}^{n+1}+\theta_{K, e}^{n} \frac{\tau p_{K}}{|K|} a_{e, K}^{n},
$$

and $\theta_{K, e}^{n}$ is some number in $(0,1)$. This proves that the entropy inequality (2.15) is satisfied for the schemes under consideration. This, together with (4.1c), proves that the $L^{\infty}$ bound (2.18) holds with $\beta=1-\gamma$.

Now, we prove the estimate (2.16). By (4.2) and (4.1c), we have

$$
\begin{aligned}
\left|v_{K, e}^{n}-v_{K_{e}, e}^{n}\right| & \leq\left|u_{K}^{n+1}-u_{K_{e}}^{n+1}\right|+2 A \frac{\tau p_{K}}{|K|} h_{K}^{\gamma} \\
& \leq\left|u_{K}^{n+1}-u_{K_{e}}^{n+1}\right|+2 A \frac{\nu}{\left\|f^{\prime}\right\|_{L^{\infty}}} h_{K}^{\gamma},
\end{aligned}
$$

and hence,

$$
\sum_{n=0}^{N_{T}-1} \sum_{K \in \mathscr{T}_{h}} \sum_{e \in \partial K}\left|v_{K, e}^{n}-v_{K_{e}, e}^{n}\right|\left|a_{K, e}^{n}\right||e| \tau \leq T_{1}+T_{2}
$$

where

$$
\begin{aligned}
& T_{1}=A \sum_{n=1}^{N_{T}} \sum_{K \in \mathscr{T}_{h}} \sum_{e \in \partial K}\left|u_{K}^{n}-u_{K_{e}}^{n}\right| h_{K}^{\gamma}|e| \tau, \\
& T_{2}=2 A^{2} \frac{\nu}{\left\|f^{\prime}\right\|_{L^{\infty}}} \sum_{n=0}^{N_{T}-1} \sum_{\substack{K \in \mathscr{T}_{h} \\
\kappa \subset \Omega(T)}} \sum_{e \in \partial K} h_{K}^{2 \gamma}|e| \tau .
\end{aligned}
$$

If $2 \gamma>1$, the term $T_{2}$ can be easily estimated by using the compactness of the support of the approximate solution and the property (2.3) of the triangulation:

$$
\begin{aligned}
T_{2} & =2 A^{2} \frac{\nu}{\left\|f^{\prime}\right\|_{L^{\infty}}} \sum_{n=0}^{N_{T}-1} \sum_{K \in \mathscr{T}_{h}, K \subset \Omega(T)} h_{K}^{2 \gamma-1}\left(p_{K} h_{K} /|K|\right)|K| \tau \\
& \leq 2 A^{2} \frac{\nu}{\left\|f^{\prime}\right\|_{L^{\infty}}} \mu T|\Omega(T)| h^{2 \gamma-1} .
\end{aligned}
$$

Estimating the term $T_{1}$ is a more delicate matter because we need to control the differences $\left|u_{K}^{n}-u_{K_{e}}^{n}\right|$. To be able to do that, we require that the values

$$
w_{K}^{n+1}=u_{K}^{n+1}+\frac{\tau}{|K|} \sum_{e \in \partial K} a_{e, K}^{n}|e|
$$

satisfy the so-called sharp entropy dissipation estimate

$$
\sum_{K \in \mathscr{T}_{h}}\left(U_{*}\left(w_{K}^{n+1}\right)-U_{*}\left(u_{K}^{n}\right)\right)|K|+C_{4} \Phi_{p}^{n} \leq 0
$$


where

$$
\Phi_{p}^{n}=\sum_{K \in \mathscr{T}_{h}} \sum_{e \in \partial K}\left|u_{K}^{n}-u_{K_{e}}^{n}\right|^{p}|e| \tau
$$

for some $p \geq 1$. In [9], it is shown indeed that (4.5) holds with $U_{*}(w)=w^{2} / 2$, and $p=2$ or $p=3$.

We start with the following result.

Lemma 4.1. Assume that $\gamma \geq 1-1 / p$. Then we have

$$
T_{1} \leq A \Theta_{p}^{1 / p}(\mu T|\Omega(T)|)^{1-1 / p} h^{\gamma-1+1 / p},
$$

where $\boldsymbol{\Theta}_{p}=\sum_{n=0}^{N_{T}-1} \Phi_{p}^{n}$.

Proof. By Hölder's inequality, we obtain easily that

$$
\begin{aligned}
T_{1} & =A \sum_{n=0}^{N_{T}-1} \sum_{K \in \mathscr{T}_{h}} \sum_{e \in \partial K}\left|u_{K}^{n}-u_{K_{e}}^{n}\right| h_{K}^{\gamma}|e| \tau \\
& \leq A \Theta_{p}^{1 / p}\left(\sum_{n=0}^{N_{T}-1} \sum_{K \in \mathscr{T}_{h}, K \subset \Omega(T)} \sum_{e \in \partial K} h_{K}^{\gamma q}|e| \tau\right)^{1 / q} \\
& =A \Theta_{p}^{1 / p}\left(\sum_{n=0}^{N_{T}-1} \sum_{K \in \mathscr{T}_{h}, K \subset \Omega(T)} h_{K}^{\gamma q} p_{K} \tau\right)^{1 / q} \\
& =A \Theta_{p}^{1 / p}\left(\sum_{n=0}^{N_{T}-1} \sum_{K \in \mathscr{T}_{h}, K \subset \Omega(T)} h_{K}^{\gamma q-1}\left(p_{K} h_{K} /|K|\right)|K| \tau\right)^{1 / q} \\
& \leq A \Theta_{p}^{1 / p}\left(\mu \sum_{n=0}^{N_{T}} \sum_{K \in \mathscr{T}_{h}, K \subset \Omega(T)}|K| \tau\right)^{1 / q} h^{\gamma-1 / q} \\
& \leq A \Theta_{p}^{1 / p}(\mu T|\Omega(T)|)^{1 / q} h^{\gamma-1 / q} .
\end{aligned}
$$

This completes the proof.

Next, we use the sharp entropy inequality (4.5) to obtain a bound on $\boldsymbol{\theta}_{p}$.

Lemma 4.2. Assume that $\gamma>\max \{1-1 / p, 1 / 2\}$. Then one has

$$
\begin{aligned}
\Theta_{p} \leq & \frac{2}{C_{4}} \sum_{K \in \mathscr{T}_{h}} U_{*}\left(u_{K}^{0}\right)|K| \\
& +\left(1-\frac{1}{p}\right) T \mu|\Omega(T)|\left(\frac{M_{*} A}{p^{1 / p} C_{4}}\right)^{q} h^{(1-1 / p)(\gamma-1+1 / p)} \\
& +2 M_{*} A^{2} \frac{\nu}{C_{4}\left\|f^{\prime}\right\|_{L^{\infty}}} \mu T|\Omega(T)| h^{2 \gamma-1} .
\end{aligned}
$$

In the case of the (first-order) monotone schemes, $A \equiv 0$, and the above inequality becomes $\Theta_{p} \leq \frac{2}{C_{4}} \sum_{K \in \mathscr{T}_{h}} U_{*}\left(u_{K}^{0}\right)|K|$, which is (up to a factor 2) the weak estimate proven for monotone schemes in [9]. In the case of the $h^{\gamma}$ antidiffusion schemes, we can say, roughly speaking, that $\boldsymbol{\Theta}_{p}$ remains bounded 
if the scheme does not produce too much antidiffusion. This is reflected in the condition $\gamma>\max \{1-1 / p, 1 / 2\}$.

Proof of Lemma 4.2. If we sum over $n$ in the sharp entropy inequality (4.5b), we get

$$
\sum_{n=1}^{N_{T}} \sum_{K \in \mathscr{T}_{h}}\left(U_{*}\left(w_{K}^{n}\right)-U_{*}\left(u_{K}^{n-1}\right)\right)|K|+C_{4} \Theta_{p} \leq 0
$$

and hence

$$
\begin{aligned}
C_{4} \boldsymbol{\Theta}_{p} & \leq \sum_{n=1}^{N_{T}} \sum_{K \in \mathscr{T}_{h}}\left(U_{*}\left(u_{K}^{n-1}\right)-U_{*}\left(w_{K}^{n}\right)\right)|K| \\
& =\sum_{n=1}^{N_{T}} \sum_{K \in \mathscr{T}_{h}}\left(U_{*}\left(u_{K}^{n-1}\right)-U_{*}\left(u_{K}^{n}\right)\right)|K|+\sum_{n=1}^{N_{T}} \sum_{K \in \mathscr{T}_{h}}\left(U_{*}\left(u_{K}^{n}\right)-U_{*}\left(w_{K}^{n}\right)\right)|K| \\
& \leq \sum_{K \in \mathscr{T}_{h}} U_{*}\left(u_{K}^{0}\right)|K|+\sum_{n=1}^{N_{T}} \sum_{K \in \mathscr{T}_{h}}\left(U_{*}\left(u_{K}^{n}\right)-U_{*}\left(w_{K}^{n}\right)\right)|K| \\
& =\sum_{K \in \mathscr{T}_{h}} U_{*}\left(u_{K}^{0}\right)|K|+\sum_{n=1}^{N_{T}} \sum_{K \in \mathscr{T}_{h}} U_{*}^{\prime}\left(\hat{v}_{K, e}^{n-1}\right)\left(u_{K}^{n}-w_{K}^{n}\right)|K|,
\end{aligned}
$$

where

$$
\hat{v}_{K, e}^{n}=\left(1-\hat{\theta}_{K, e}^{n}\right) u_{K, e}^{n+1}+\hat{\theta}_{K, e}^{n} w_{K, e}^{n+1}=u_{K, e}^{n+1}+\hat{\theta}_{K, e}^{n} \frac{\tau p_{K}}{|K|} a_{e, K}^{n},
$$

and $\hat{\theta}_{K, e}^{n}$ is some number in $(0,1)$. Then,

$$
\begin{aligned}
C_{4} \Theta_{p} & \leq \sum_{K \in \mathscr{T}_{h}} U_{*}\left(u_{K}^{0}\right)|K|-\sum_{n=1}^{N_{T}} \sum_{K \in \mathscr{T}_{h}} \sum_{e \in \partial K} U_{*}^{\prime}\left(\hat{v}_{K, e}^{n-1}\right) a_{e, K}^{n-1}|e| \tau \\
& =\sum_{K \in \mathscr{T}_{h}} U_{*}\left(u_{K}^{0}\right)|K|-\sum_{n=1}^{N_{T}} \sum_{K \in \mathscr{T}_{h}} \sum_{e \in \partial K}\left(U_{*}^{\prime}\left(\hat{v}_{K, e}^{n-1}\right)-U_{*}^{\prime}\left(\hat{v}_{e}^{n-1}\right)\right) a_{e, K}^{n-1}|e| \tau,
\end{aligned}
$$

where $\hat{v}_{e}^{n}=\left(\hat{v}_{K, e}^{n}+\hat{v}_{K_{e}, e}^{n}\right) / 2$. Hence, with $M_{*}=\sup _{u \in \mathbb{R}}\left|U_{*}^{\prime \prime}(u)\right|$, which by hypothesis is a constant, we have

$$
C_{4} \Theta_{p} \leq \sum_{K \in \mathscr{I}_{h}} U_{*}\left(u_{K}^{0}\right)|K|+\frac{1}{2} M_{*} \sum_{n=0}^{N_{T}-1} \sum_{K \in \mathscr{T}_{h}} \sum_{e \in \partial K}\left|\hat{v}_{K, e}^{n}-\hat{v}_{K_{e}, e}^{n}\right|\left|a_{K, e}^{n}\right||e| \tau .
$$

By (4.2) and (4.1c), we have

$$
C_{4} \Theta_{p} \leq \sum_{K \in \mathscr{T}_{h}} U_{*}\left(u_{K}^{0}\right)|K|+\frac{1}{2} M_{*}\left\{T_{1}+T_{2}\right\}
$$

and, by (4.4a) and Lemma 4.1,

$$
\begin{gathered}
C_{4} \Theta_{p} \leq \sum_{K \in \mathscr{T}_{h}} U_{*}\left(u_{K}^{0}\right)|K|+M_{*} A^{2} \frac{\nu}{\left\|f^{\prime}\right\|_{L^{\infty}}} \mu T|\Omega(T)| h^{2 \gamma-1} \\
+\frac{1}{2} M_{*} A \Theta_{p}^{1 / p}(\mu T|\Omega(T)|)^{1-1 / p} h^{\gamma-1+1 / p}
\end{gathered}
$$


By using the inequality

$$
a b \leq \frac{C_{4}}{2} a^{p}+\left(1-\frac{1}{p}\right)\left(\frac{2}{p C_{4}}\right)^{q / p} b^{q},
$$

with $a=\Theta_{p}$, we get, after some simple algebraic manipulations,

$$
\begin{aligned}
C_{4} \Theta_{p} \leq & 2 \sum_{K \in \mathscr{T}_{h}} U_{*}\left(u_{K}^{0}\right)|K| \\
& +\left(1-\frac{1}{p}\right) T \mu|\Omega(T)|\left(\frac{M_{*} A}{p^{1 / p} C_{4}^{1 / p}}\right)^{q} h^{q(\gamma-1+1 / p)} \\
& +2 M_{*} A^{2} \frac{\nu}{\left\|f^{\prime}\right\|_{L^{\infty}}} \mu T|\Omega(T)| h^{2 \gamma-1}
\end{aligned}
$$

This completes the proof.

In this way, by Lemmas 4.1 and 4.2 , if $\gamma>\max \{1-1 / p, 1 / 2\}$, then

$$
T_{1} \leq C_{5} h^{\gamma-1+1 / p} \text {. }
$$

Finally, by (4.4a) and (4.4b), we get that the scheme under consideration satisfies the estimate $(2.16)$ with

$$
\alpha=\min \{2 \gamma-1, \gamma-1+1 / p\} .
$$

Since $\beta=1-\gamma$, Theorem 2.1 states that the scheme under consideration converges to the entropy solution, with an order of convergence no smaller than

$$
\min \{\alpha, 1-\beta\} / 2=\min \{\gamma-1 / 2, \gamma / 2-1 / 2+1 / 2 p, \gamma / 2\} .
$$

Taking $\gamma=1$, we see that the order of convergence is no smaller than $1 / 2 p$.

\section{ACKNOWLEDGMENTS}

The first author would like to thank Guangshan Jiang and Chi-Wang Shu for their comments that led to a better presentation of this paper. This paper was completed when the third author was a Courant Instructor at the Courant Institute of Mathematical Sciences at New York University, supported by NSF grant DMS-88-06731. The third author would like to thank Gui-Qiang Chen, Jonathan Goodman, Peter Lax, Jian-Guo Liu, Richard Sanders, and Eitan Tadmor for fruitful conversations on the content of this paper.

\section{BIBLIOGRAPHY}

1. G.-Q. Chen, Q. Du, and E. Tadmor, Spectral viscosity approximation to multidimensional scalar conservation laws, Math. Comp. 61 (1993), 629-643.

2. G.-Q. Chen and Ph. LeFloch, Entropy flux-splittings for hyperbolic conservation laws. Part 1: general framework, submitted to Comm. Pure Appl. Math., in preparation.

3. $\ldots$, Entropy flux-splittings for hyperbolic conservation laws. Part 2: Gas dynamics equations, in preparation.

4. I.L. Chern, Stability theorem and truncation error analysis for the Glimm scheme and for a front tracking method for flows with strong discontinuities, Comm. Pure Appl. Math. 42 (1989), 815-844.

5. B. Cockburn, The quasi-monotone schemes for scalar conservation laws. I, SIAM J. Numer. Anal. 26 (1989), 1325-1341. 
6. $\ldots$ The quasi-monotone schemes for scalar conservation laws. II, SIAM J. Numer. Anal. 27 (1990), 247-258.

7. 27 (1990), 259-276.

8. On the continuity in $B V(\Omega)$ of the $L^{2}$-projection into finite element spaces, Math. Comp. 57 (1991), 551-561.

9. B. Cockburn, F. Coquel and $\mathrm{Ph}$. LeFloch, Convergence of finite volume methods for multidimensional conservation laws, SIAM J. Numer. Anal. (to appear).

10. B. Cockburn and C.-W. Shu, TVB Runge-Kutta local projection discontinuous Galerkin finite element method for conservation laws II: general framework, Math. Comp. 52 (1989), 411-435.

11. B. Cockburn, S.-C. Hou, and C.-W. Shu, The Runge-Kutta local projection discontinuous Galerkin finite element method for conservation laws IV: the multidimensional case, Math. Comp. 54 (1990), 545-581.

12. B. Cockburn and C.-W. Shu, The Runge-Kutta local projection $P^{1}$ discontinuous Galerkin finite element method for scalar conservation laws, ICASE Report No. 91-32, 1991.

13. F. Coquel and $\mathrm{Ph}$. LeFloch, Convergence of finite difference schemes for conservation laws in several space dimensions, C. R. Acad. Sci. Paris, Série I, 31017 (1990), 455-460.

14. __ Convergence of finite difference schemes for conservation laws in several space dimensions: the corrected antidiffusive flux approach, Math. Comp. 57 (1991), 169-210.

15. __ Convergence of finite difference schemes for conservation laws in several space dimensions: a general theory, SIAM J. Numer. Anal. 30 (1993), 675-700.

16. M. Crandall and A. Majda, Monotone difference approximations for scalar conservation laws, Math. Comp. 34 (1980), 1-21.

17. R.J. DiPerna, Convergence of approximate solutions to conservation laws, Arch. Rational Mech. Anal. 82 (1983), 27-70.

18. _ Measure-valued solutions to conservations laws, Arch. Rational Mech. Anal. 88 (1985), 223-270.

19. J.B. Goodman and R.J. LeVeque, On the accuracy of stable schemes for $2 D$ scalar conservation laws, Math. Comp. 45 (1985), 15-21.

20. A. Harten, J.M. Hyman, and P.D. Lax, On finite-difference approximations and entropy conditions for shocks, Comm. Pure Appl. Math. 29 (1976), 297-322.

21. A. Harten, P.D. Lax, and B. van Leer, On upstream differencing and Godunov-type schemes for hyperbolic conservation laws, SIAM Rev. 25 (1983), 35-61.

22. D. Hoff and J.S. Smoller, Error bounds for the Glimm scheme for a scalar conservation law, Trans. Amer. Math. Soc. 289 (1988), 611-642.

23. T.Y. Hou and Ph.G. LeFloch, Why nonconservative schemes converge to wrong solutions: error analysis, Math. Comp. 62 (1994), 497-530.

24. C. Johnson and A. Szepessy, On the convergence of a finite element method for a nonlinear hyperbolic conservation law, Math. Comp. 49 (1988), 427-444.

25. __ A posteriori error estimate for a finite element method, Preprint (1993).

26. S.N. Kružkov, First order quasilinear equations in several independent variables, Math. USSR Sb. 10 (1970), 217-243.

27. _ On the methods of construction of the general solution of the Cauchy problem for first order quasilinear equations, Uspehi Mat. Nauk 20 (1965), 112-118. (Russian)

28. N.N. Kuznetsov, Accuracy of some approximate methods for computing the weak solutions of a first-order quasi-linear equation, USSR Comput. Math. and Math. Phys. 16 (1976), 105-119.

29. __ On stable methods for solving nonlinear first-order partial differential equations in the class of discontinuous solutions, Topics in Numerical Analysis III (Proc. Roy. Irish Acad. Conf.), Trinity College, Dublin, 1976, pp. 183-192.

30. P.D. Lax, Hyperbolic systems of conservation laws II, Comm. Pure Appl. Math. 10 (1957), 537-566. 
31. Hyperbolic systems of conservation laws and the mathematical theory of shock waves, SIAM, Philadelphia, PA, 1973.

32. $\mathrm{Ph}$. LeFloch, Convergence des méthodes de volumes finis monotones pour les lois de conservation scalaires, Communication to Ecole CEA-EDF-INRIA, Meeting on finite volume methods, October 1992 (unpublished notes).

33. Ph. LeFloch and J.G. Liu, Entropy and monotonicity consistent EMO schemes for conservation laws, in preparation.

34. B.J. Lucier, Error bounds for the methods of Glimm, Godunov and LeVeque, SIAM J. Numer. Anal. 22 (1985), 1074-1081.

35. _ A moving mesh numerical method for hyperbolic conservation laws, Math. Comp. 46 (1986), 59-69.

36. 47 (1986), 19-36.

37. S. Nessyahu and E. Tadmor, The convergence rate of approximate solutions for nonlinear scalar conservation laws, SIAM J. Numer. Anal. 29 (1992), 1-15.

38. S. Osher, Riemann solvers, the entropy condition and difference approximations, SIAM J. Numer. Anal. 21 (1984), 217-235.

39. S. Osher and R. Sanders, Numerical approximations to nonlinear conservation laws with locally varying time and space grids, Math. Comp. 41 (1983), 321-336.

40. S. Osher and $\mathrm{E}$. Tadmor, On the convergence of difference approximations to scalar conservation laws, Math. Comp. 50 (1988), 19-51.

41. R. Sanders, On convergence of monotone finite difference schemes with variable spatial differencing, Math. Comp. 40 (1983), 91-106.

42. __ Finite difference techniques for nonlinear hyperbolic conservation laws, Lectures in Appl. Math., vol. 22, Amer. Math. Soc., Providence, RI, 1985, pp. 209-220.

43. J.S. Smoller, Shock waves and reaction diffusion equations, Springer-Verlag, New York, 1983.

44. A. Szepessy, Convergence of a shock-capturing streamline diffusion finite element method for scalar conservation laws in two space dimensions, Math. Comp. 53 (1989), 527-545.

45. __ Convergence of a streamline diffusion finite element method for a conservation law with boundary conditions, RAIRO Modél. Math. Anal. Numér. 25 (1991), 749-783.

46. E. Tadmor, Numerical viscosity and the entropy condition for conservative difference schemes, Math. Comp. 43 (1984), 369-382.

47. __ Semi-discrete approximations to nonlinear systems of conservation laws; consistency and $L^{\infty}$ imply convergence, ICASE Report 88-41 (1988).

48. __ Local error estimates for discontinuous solutions of nonlinear hyperbolic equations, SIAM J. Numer. Anal. 28 (1991), 891-906.

50. J.P. Vila, Problèmes nonlinéaires appliqués, Ecoles CEA-EDF-INRIA, Clamart, France, 1993.

51. A.I. Volpert, The space BV and quasilinear equations, Math. USSR Sb. 2 (1967), 257-267.

School of Mathematics, University of Minnesota, 127 Vincent Hall, Minneapolis, MinNESOTA 55455

E-mail address: cockburn@math.umn.edu

ONERA, 29 av. De la Division Leclerc, BP 72, F-92322 Chatillon Cedex, France

E-mail address: coquel@onera.fr

Courant Institute of Mathematical Sciences, New York University, 251 Mercer Street, New York, New York 10012

E-mail address: lefloch@cmapx.polytechnique.fr 